\title{
A Separation Logic for Negative Dependence
}

\author{
JIALU BAO, Cornell University, USA \\ MARCO GABOARDI, Boston University, USA \\ JUSTIN HSU, Cornell University, USA \\ JOSEPH TASSAROTTI, Boston College, USA
}

\begin{abstract}
Formal reasoning about hashing-based probabilistic data structures often requires reasoning about random variables where when one variable gets larger (such as the number of elements hashed into one bucket), the others tend to be smaller (like the number of elements hashed into the other buckets). This is an example of negative dependence, a generalization of probabilistic independence that has recently found interesting applications in algorithm design and machine learning. Despite the usefulness of negative dependence for the analyses of probabilistic data structures, existing verification methods cannot establish this property for randomized programs.

To fill this gap, we design LINA, a probabilistic separation logic for reasoning about negative dependence. Following recent works on probabilistic separation logic using separating conjunction to reason about the probabilistic independence of random variables, we use separating conjunction to reason about negative dependence. Our assertion logic features two separating conjunctions, one for independence and one for negative dependence. We generalize the logic of bunched implications (BI) to support multiple separating conjunctions, and provide a sound and complete proof system. Notably, the semantics for separating conjunction relies on a non-deterministic, rather than partial, operation for combining resources. By drawing on closure properties for negative dependence, our program logic supports a Frame-like rule for negative dependence and monotone operations. We demonstrate how LINA can verify probabilistic properties of hash-based data structures and balls-into-bins processes.
\end{abstract}

\section{CCS Concepts: • Theory of computation $\rightarrow$ Separation logic.}

Additional Key Words and Phrases: Probabilistic programs, separation logic, negative dependence

\section{ACM Reference Format:}

Jialu Bao, Marco Gaboardi, Justin Hsu, and Joseph Tassarotti. 2022. A Separation Logic for Negative Dependence. Proc. ACM Program. Lang. 6, POPL, Article 57 (January 2022), 29 pages. https://doi.org/10.1145/3498719

\section{INTRODUCTION}

Hashing plays a fundamental role in many probabilistic data structures, from basic hash tables to more sophisticated schemes such as Bloom filters. In these applications, a hash function $h$ maps a universe of possible values, typically large, to a set of buckets, typically small. Hash-based data structures satisfy a variety of probabilistic guarantees. For instance, we may be interested in the false positive rate: the probability that a data structure mistakenly identifies an element as being stored in a collection, when it was not inserted. We may also be interested in load measures, such as the probability that a bucket in the data structure overflows. A typical way to analyze these quantities is to treat random hash functions as balls-into-bins processes. For example, hashing $N$

Authors' addresses: Jialu Bao, Cornell University, USA; Marco Gaboardi, Boston University, USA; Justin Hsu, Cornell University, USA; Joseph Tassarotti, Boston College, USA.

This work is licensed under a Creative Commons Attribution 4.0 International License.

(c) 2022 Copyright held by the owner/author(s).

2475-1421/2022/1-ART57

https://doi.org/10.1145/3498719

Proc. ACM Program. Lang., Vol. 6, No. POPL, Article 57. Publication date: January 2022. 
unique elements into $B$ bins can be modeled as throwing $N$ balls into $B$ bins, where each bin is drawn uniformly at random.

While this modeling is convenient, one complication is that the counts of the elements in the different buckets are not probabilistically independent: one bin containing many elements makes it more likely that other bins contain few elements. The lack of independence makes it difficult to reason about multiple bins, for instance bounding the number of empty bins. Moreover, many common tools for analyzing probabilistic processes, like concentration bounds, usually require independence. This subtlety has also been a source of problems in pen-and-paper analyses of probabilistic data structures. For instance, the standard analysis of the Bloom filter bounds the number of occupied bins in order to bound the false positive rate. The original version of this analysis presented by Bloom [1970], and also repeated in many papers, assumes that the bin counts are independent. However, Bose et al. [2008] pointed out that this assumption is incorrect, and in fact the claimed upper-bound on the false-positive rate is actually a lower bound. Proving a correct bound on the false-positive rate required a substantially more complicated argument; recently, Gopinathan and Sergey [2020] mechanized a correct, but complex proof in Coq.

We aim to develop a simpler method to formally reason about hash-based data structures and balls-into-bins processes, drawing on a key concept in probability theory: negative dependence.

Towards a simpler analysis: negative dependence. To study balls-into-bins processes and other phenomena, researchers in probability theory have developed a theory of negative dependence [Pemantle 2000]. Intuitively, variables are negatively dependent if when one is larger, then the others tend to be smaller. The counts of the bins in the balls-into-bins process is a motivating example of negative dependence.

While there are multiple incomparable definitions of negative dependence, Joag-Dev and Proschan [1983] proposed a notion called negative association (NA) that has many good probabilistic properties. For instance, the bins' counts in the balls-into-bins process satisfies NA, and NA's closure properties enable simple, calculation-free proofs of NA. More intriguingly, as Dubhashi and Ranjan [1998] identified, sums of NA variables satisfy some concentration bounds that usually assume probabilistic independence, including the widely-used Chernoff bounds.

Our goal: formal reasoning about negative dependence. From a verification perspective, the closure properties suggest a compositional method for proving NA in probabilistic programs. In this work, we develop a separation logic for negative dependence, building on a separation logic for probabilistic programs called PSL [Barthe et al. 2020]. Like all separation logics, PSL is a program logic where assertions are drawn from the logic of bunched implications (BI) [O'Hearn and Pym 1999], a substructural logic. In PSL, the separating conjunction $*$ states that two sets of variables are probabilistically independent, a common and useful property when analyzing probabilistic programs.

We aim to extend the assertions of PSL so that they can describe both independence and negative dependence. There are three main difficulties:

- To support reasoning about negative dependence, the assertion logic needs to be extended with a second separating conjunction that is weaker than the separating conjunction of PSL. It is easy to extend the syntax of formulas, but the extended logic should also enjoy good metatheoretical properties like BI does, including a sound and complete proof system.

- The standard resource semantics of BI [Pym 2002], based on partial commutative monoids (PCMs), is not expressive enough to model negative association because two variables with given marginal distributions can be negatively associated in more than one way. 
- Defining the semantics of separating conjunction to capture NA is surprisingly challenging. Straightforward definitions fail to satisfy expected properties, like associativity of separating conjunction.

Beyond the assertions, it is also unclear how to integrate negative association with the proof rules of PSL. In particular, to view negative association as a kind of separation, our program logic should have an analogue of the Frame rule for NA.

Contributions and outline. In this paper, we offer the following contributions.

- A novel logic $M$-BI that extends BI with multiple separating conjunctions, related by a preorder. Following Docherty [2019], models of $M$-BI allow two states to be combined into a single state in more than one way (Section 3). We develop a proof system for $M$-BI, and use Docherty's duality-theoretic approach to prove soundness and completeness.

- A probabilistic model of $M$-BI that can capture both the independence and negative association (Section 4). There are two interesting aspects of our model:

- We crucially use the "non-deterministic" combination of resources allowed by Docherty's semantics of BI. While this semantics was originally used to simplify the metatheory of BI, our model shows that the added flexibility can enable new applications of the logic.

- Our model relies on a novel notion called PNA that is more expressive than Joag-Dev and Proschan [1983]'s NA. The generalization is needed to satisfy the conditions for an $M-\mathrm{BI}$ model. Moreover, the closure properties and useful consequences of NA continue to hold for our generalization.

- A program logic, LINA (Logic of Independence and Negative Association), extending PSL with $M$-BI-assertions and a new negative-association Frame rule (Section 5). Being a conservative extension of PSL, the proof rules of PSL remain valid in LINA. We demonstrate our program logic by proving negative association and related properties on several case studies (Section 6). For example, using NA, it is possible to give a significantly simpler verification of the false positive rate of the Bloom filter. Another example-an analysis of a repeated ballsinto-bins process motivated by distributed computing-involves a loop with a probabilistic guard, and requires reasoning about conditional distributions.

We discuss related work in Section 7, and conclude in Section 8.

\section{OVERVIEW AND KEY IDEA}

In this section, we introduce negative association as a tool for analyzing hashing-based algorithms. We use Bloom filters, a hash-based data structure, as a motivating example. After sketching a standard proof applying negative association to Bloom filters, we will show how the same analysis can be formalized in LINA.

\subsection{Background on Negative Association}

Negative association is a property of a set of random variables, which intuitively says that when some variables are larger, we expect the others to be smaller. It is formalized as follows:

Definition 2.1 (Negative Association (NA)). Let $X_{1}, \ldots X_{n}$ be random variables. The set $\left\{X_{i}\right\}_{i}$ is negatively associated (NA) if for every pair of subsets $I, J \subseteq\{1, \ldots, n\}$ such that $I \cap J=\emptyset$, and every pair of both monotone or both antitone functions ${ }^{1} f: \mathbb{R}^{|I|} \rightarrow \mathbb{R}$ and $g: \mathbb{R}^{|J|} \rightarrow \mathbb{R}$, where $f, g$ is either lower bounded or upper bounded, we have:

$$
\mathbb{E}\left[f\left(X_{i}, i \in I\right) \cdot g\left(X_{j}, j \in J\right)\right] \leq \mathbb{E}\left[f\left(X_{i}, i \in I\right)\right] \cdot \mathbb{E}\left[g\left(X_{j}, j \in J\right)\right]
$$

\footnotetext{
${ }^{1}$ In the following, we will consistently use monotone to mean monotonically non-decreasing and antitone to mean monotonically non-increasing.
} 
We can view NA as generalizing independence: a set of independent random variables is NA because equality holds. NA also strengthens negative covariance, a simpler notion of negative dependence that requires $\mathbb{E}\left[\prod_{i \in[n]} X_{i}\right] \leq \prod_{i \in[n]} \mathbb{E}\left[X_{i}\right]$.

The survey paper by Dubhashi and Ranjan [1998] explains several properties of NA random variables useful for algorithm analysis. First, some standard theorems about sums of independent random variables apply more generally to sums of NA random variables. In particular, the widelyused Chernoff bound, which intuitively says that the sum of independent random variables is close to the expected value of the sum with high probability, holds also for NA variables. In addition, NA is preserved by some common operations on random variables. Thus, we can easily prove that a set of random variables satisfies NA if they are generated by applying NA-preserving operations to a few basic, building-block random variables:

THEOREM 2.2. The random variables $\left\{X_{i}\right\}_{i}$ in the following cases are negatively associated:

(1) Let $\left\{X_{1}, \ldots, X_{n}\right\}$ be Bernoulli random variables such that $\sum X_{i}=1$.

(2) Let $X_{i}$ be the $i$-th entry in the vector $X$, where $X$ is a uniformly random permutation of a finite, nonempty set $A$.

(3) Let $\left\{X_{1}, \ldots, X_{n}\right\}$ be independent random variables.

In particular, the first case of this theorem implies that if we draw a length- $n$ one-hot vector, i.e., a vector that has one entry being one and all remaining entries being zero, uniformly at random, then the entries of the vector satisfy negative association.

The following theorem states two key closure properties of NA random variables.

THEOREM 2.3. The set $S$ of random variables in the following cases are negatively associated:

(1) Let $T$ be negatively associated, and let $S$ be a non-empty subset of $T$.

(2) Let $T$ and $U$ be two sets of negatively associated random variables such that every $X \in T$ and $Y \in U$ is independent of each other. Let $S=T \cup U$.

(3) Let $\left\{X_{1}, \ldots X_{n}\right\}$ be negatively-associated, and $I_{1}, \ldots, I_{m}$ be a partition of the set $\{1, \ldots, n\}$. For each $1 \leq j \leq m$, let $f_{j}: \mathbb{R}^{\left|I_{j}\right|} \rightarrow \mathbb{R}$ be monotone. Let $S=\left\{f_{1}\left(X_{k}, k \in I_{1}\right), \ldots, f_{m}\left(X_{k}, k \in I_{m}\right)\right\}$.

The first case shows that negative association is preserved if we discard random variables, while the second case allows us to join two independent sets of negatively associated random variables to form a larger negatively associated set. Finally, the third case guarantees that negative association is preserved under applying monotone maps on disjoint subsets of variables.

\subsection{Example: Bloom Filters}

We demonstrate how NA and its closure properties can be used to analyze Bloom filters. A Bloom filter is a space-efficient probabilistic data structure for storing a set of items from a universe $U$. An $N$-bit Bloom filter consists of a length- $N$ array bloom holding zero-one entries. We assume there is a family $\mathcal{A}$ of hash functions mapping $U$ to $\{0, \ldots, N-1\}$ such that for any $x \in U$ and any bucket $k, \mathbb{P}_{f \in \mathcal{A}}(f(x)=k)=1 / N$. Let $l_{1}, \ldots, l_{H}$ be a collection of hash functions drawn from $\mathcal{A}$. We assume the hash functions are independent, meaning the collection of variables $\left\{l_{i}(x) \mid x \in U, i \in\{1, \ldots, H\}\right\}$ are mutually independent. To add an item $x \in U$ to the filter, we compute $l_{1}(x), \ldots, l_{H}(x)$ to get $H$ positions in the bit array and then set the bits at each of these positions to 1 . To check if an item $y$ is in the filter, we check whether the bits at positions $l_{1}(y), \ldots, l_{H}(y)$ in bloom are all 1 . If they are, the item is said to be in the filter, but if any is 0 , then the item is not in the filter. This membership test may suffer from false positives, i.e., it may show that an item $y$ is in the filter even when $y$ was never added to the filter. This can happen because with hash collisions, other items added to the Bloom filter could set all the bits at locations 
$l_{1}(y), \ldots, l_{H}(y)$ to 1 . A basic quantity of interest is the false positive rate: the probability that a Bloom filter reports a false positive.

Our goal is to bound an $N$-bit Bloom filter's false positive rate after $M$ distinct items are added, assuming that it uses $H$ independent hash functions. Here, we briefly sketch a standard proof where negative association plays a key role. Let $x$ be some data item not in the set, and let FP be the event that the Bloom filter returns a false positive on $x$. We split the analysis of the probability of FP into two steps.

For the first step, we condition on $\rho$, the fraction of bits in bloom that are set to 1 after all items have been added. With $\rho$ fixed to some value, for each hash function $l_{\alpha}$ the probability that bloom $\left[l_{\alpha}(x)\right]=1$ is $\rho$. For $x$ to be a false positive, we must have bloom $\left[l_{\alpha}(x)\right]=1$ for all $\alpha$. Since $l_{1}, \ldots, l_{H}$ are independent hash functions, this occurs with probability $\rho^{H}$.

The next step is to show that with high probability, $\rho$ lies within a narrow range around its expected value. Before showing how to prove this, let us first see why such a bound is useful. Let $\mu$ be the expected value of $\rho$. Suppose we have that for some small $\epsilon$ and $\delta$ that $\operatorname{Pr}[\rho \leq \mu+\epsilon] \geq 1-\delta$. Then, by the law of total probability we have:

$$
\begin{aligned}
\operatorname{Pr}[\mathrm{FP}] & \leq \operatorname{Pr}[\mathrm{FP} \mid \rho \leq \mu+\epsilon]+\operatorname{Pr}[\rho>\mu+\epsilon] \\
& \leq(\mu+\epsilon)^{H}+\delta
\end{aligned}
$$

where the second line follows from the calculation of the probability of FP when conditioned on $\rho$.

Now, we turn to the question of how to obtain a bound of the form $\operatorname{Pr}[\rho \leq \mu+\epsilon]<1-\delta$. As mentioned in Section 1, a common (incorrect) analysis of $\rho$ assumes that the entries of bloom are independent, and then applies a Chernoff bound. However, the entries in bloom, $\{\text { bloom }[\beta]\}_{\beta}$, are not independent-what we can actually prove is that are negatively associated, which fortunately still allows us to apply the Chernoff bound, as stated later in Theorem 6.1.

To see that the $\{\text { bloom }[\beta]\}_{\beta}$ are NA, consider the program in Figure 1, which models the process of adding $M$ distinct items to the Bloom filter. Because the $M$ items are distinct, we model the hash functions as independently, randomly sampling hash values for each item as they are added, a standard model used in the analysis of hashing data structures [Mitzenmacher and Upfal 2005]. That is, we encode the hashing step as sampling a one-hot vector with the command oh and storing it in the variable bin, where the hot bit of the vector bin represents the selected position. To set the corresponding position in the filter to 1, we update bloom to be bloom $\|$ bin, the bitwise-or of the current array and the sampled onehot array. To show that $\{\text { bloom }[\beta]\}_{\beta}$ are NA, we can reason using the closure properties. Initially, bloom is set to zero $(N)$. Any set of

$$
\begin{aligned}
& \text { bloom } \leftarrow \text { zero }(N) ; \\
& m \leftarrow 0 ; \\
& \text { while } m<M \text { do } \\
& h \leftarrow 0 \\
& \text { while } h<H \text { do } \\
& \quad \text { bin } \$ \text { oh }([N]) ; \\
& \quad \text { upd } \leftarrow \text { bloom } \| \text { bin; } \\
& \quad \text { bloom } \leftarrow \text { upd; } \\
& \quad h \leftarrow h+1 ; \\
& m \leftarrow m+1
\end{aligned}
$$

Fig. 1. Example: Bloom filter constant random variables is independent, and hence negatively associated by Theorem 2.3. Next, when an item is added, the bin array is NA by Theorem 2.2. Because bin is sampled independently of bloom, the set $\{\text { bloom }[\beta]\}_{\beta} \cup\{\operatorname{bin}[\beta]\}_{\beta}$ is NA. The bitwise-or operation \| is monotone, so again by Theorem 2.3, the array upd is negatively associated, thereby showing that bloom is NA at the end of each loop iteration.

\subsection{Representing Negative Association with Separating Conjunction}

Now that we have seen some properties of negative association and how they can be used to analyze the Bloom filter, we give a high-level explanation of how these ideas are formalized in LINA, a novel program logic that is a core contribution of our work. As mentioned in Section 1, an 
earlier separation logic PSL has a separating conjunction $*$ which is interpreted as probabilistic independence. That is, a program state satisfies $P * Q$ if its randomized program variables can be split so that one subset satisfies $P$, another satisfies $Q$, and the distributions of these two sets are independent. LINA augments PSL with a weaker separating conjunction $\circledast$ modeling negative association. The precise definition of negative association needs to be modified to form a proper model of bunched implications, but for now, one can informally think of $P \circledast Q$ as meaning the random variables can be split into two sets negatively associated with each other that satisfy $P$ and $Q$ respectively.

The proof rules of LINA can be used to prove NA by applying closure properties to building-block NA-distributions, much like in our proof sketch above for the Bloom filter. For example, we can derive a rule that captures NA of entries in a one-hot distribution:

$$
\vdash\{\top\} x \stackrel{\infty}{o h}(n)\{\overbrace{\beta=0}^{n}\langle x[\beta]\rangle\},
$$

where the assertion $\langle y\rangle$ means that the program variable $y$ is distributed according to some unspecified probability distribution, and $\circledast$ is an iterated version of the $\circledast$ separating conjunction. Thus, the post-condition here says that all of the entries of the $x$ vector are negatively associated.

Meanwhile, since NA is closed under monotone maps, we obtain a form of separation logic's frame rule for $\circledast$ :

$\frac{\vdash\{\phi\} c\{y \sim f(X)\} \quad f \text { monotone } \quad \text { (side conditions omitted) }}{\vdash\{\phi \circledast \eta\} c\{\langle y\rangle \circledast \eta\}}$,

where $X$ is a set of variables contained in any program states satisfying $\phi, f$ is a monotone function mapping $X$ to a variable $y$, and $\eta$ is an assertion on some other random variables that are negatively associated with those satisfying $\phi$. (We describe a complete version of this rule with all side conditions later, in Section 5.4.) Using this rule, we can show monotone vector operations like \| in the Bloom filter example preserve negative associativity. For example, we can derive:

$$
\vdash\left\{\circledast_{\beta=0}^{n}\langle x[\beta]\rangle \circledast \circledast_{\gamma=0}^{n}\langle y[\gamma]\rangle\right\} z \leftarrow x \| y\left\{\bigoplus_{\beta=0}^{n}\langle z[\beta]\rangle\right\},
$$

which says that if the union of entries in $x$ and entries in $y$ satisfy NA, then entries in $z=x \| y$ also satisfy NA.

We now sketch how to formalize the proof that $\{\text { bloom }[\beta]\}_{\beta}$ in the Bloom filter are NA; we defer the rest of the proof of this example to Section 6. The basic idea is to establish $\circledast_{\beta=0}^{N}\langle$ bloom $[\beta]\rangle$ as a loop invariant. When an item is added in the loop, we combine the frame rule with the one-hot sampling vector rule to get that the bin vector is negatively associated, thus showing: $\circledast_{\beta=0}^{N}\langle\operatorname{bloom}[\beta]\rangle \circledast \circledast_{\gamma=0}^{N}\langle\operatorname{bin}[\gamma]\rangle$. Applying the rule for $\|$ above, we obtain that upd is negatively associated, $\circledast_{\beta=0}^{N}\langle u p d[\beta]\rangle$. At that point upd is assigned to bloom, restoring the loop invariant.

\section{THE LOGIC $M$-BI}

Having seen the role of negative association in analyzing randomized algorithms and how its properties correspond to rules in LINA, we now show how negative association can be interpreted by separating conjunction. As a first step, we extend the logic of bunched implications (BI), the assertion logic underlying separation logic, to support multiple forms of separating conjunction simultaneously, related by a pre-order. Our motivation to design this logic is to reason about 
independence and negative association in one logic and capture that independence implies negative association, but the logic is more general and accommodates other potentially interesting models.

\subsection{The Syntax and Proof Rules}

Let $\mathcal{A P}$ be a set of atomic propositions, and $(M, \leq)$ be a finite pre-order. The formula in the logic of $M$-bunched implications ( $M$-BI) has the following grammar:

$$
P, Q::=p \in \mathcal{A P}|\top| I_{m \in M}|\perp| P \wedge Q|P \vee Q| P \rightarrow Q\left|P *_{m \in M} Q\right| P *_{m \in M} Q .
$$

$M$-BI associates each element of $M$ with a separating conjunction $*_{m}$, a corresponding multiplicative identity $I_{m}$ and a separating implication $*_{m}$. The proof system for M-BI is based on the proof system for BI, with indexed copy of rules for each separation, and in addition has *-WeAKenING rules. We present the full Hilbert-style proof system in the extended version [Bao et al. 2022]; most of the rules are the same as in the proof system for BI. Here, we only comment on the new rules.

The $*$-Weakening rule says that if $m_{1} \leq m_{2}$, then the assertion $P * m_{1} Q$ implies $P * m_{2} Q$.

$$
\frac{m_{1} \leq m_{2}}{P *_{m_{1}} Q \vdash P *_{m_{2}} Q} * \text {-WEAKENING }
$$

We can derive analogous weakening rules for separating implications and multiplicative identities, in the reverse direction.

Lemma 3.1. The following rules are derivable in M-BI:

$$
\frac{m_{1} \leq m_{2}}{P * m_{2} Q \vdash P * m_{1} Q} *-\text { WEAKENING } \quad \frac{m_{1} \leq m_{2}}{I_{m_{2}} \vdash I_{m_{1}}} U_{N I T W E A K E N I N G}
$$

\subsection{Semantics}

As is standard with bunched logics [Pym et al. 2004], we give a Kripke style semantics to $M$-BI. We will define a structure called $M$-BI frame, and then define $M$-BI models and the satisfaction rules on $M$-BI models.

An $M$-BI frame is a collection of BI frames satisfying some frame conditions. While BI frames are often presented as partial, pre-ordered commutative monoids over states, we need the more general presentation due to Docherty [2019], where the binary operation returns a set of states, instead of at most one state. Such binary operations can be deterministic (returning a set of at most one element) or non-deterministic. The admission of non-deterministic models was originally motivated by the metatheory; somewhat surprisingly, it is also a crucial ingredient in defining the negative association model we will see in Section 4.

Definition 3.2 (BI Frame). A (Down-Closed) BI frame is a structure $X=(X, \sqsubseteq, \oplus, E)$ such that $\sqsubseteq$ is a pre-order on the set of states $X, \oplus: X^{2} \rightarrow \mathcal{P}(X)$ is a binary operation, and $E \subseteq X$, satisfying following frame conditions (with outermost universal quantification omitted for readability):

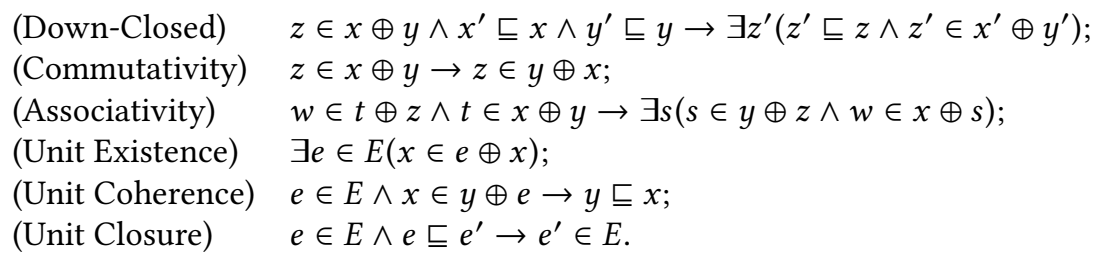

Since all frames we consider in this paper will be Down-Closed, we often abbreviate Down-Closed BI frame as just BI frame. The (Commutativity), (Associativity) and (Unit Existence) conditions capture the properties of commutative monoids, and the (Down-Closed) condition ensures that the 
binary operation is coherent with the pre-order. Properties (Associativity) and (Commutativity) are generalizations of the usual algebraic properties to accommodate the non-determinism. The three Unit frame conditions ensure that the set $E$ behaves like a set of units, and satisfies various closure properties under the binary operation and the pre-order.

We can now define $M$-BI frame to be a collection of BI frames sharing the same set of states and pre-order, with ordered binary operations:

Definition 3.3 (M-BI Frame). An $M$-BI frame is a structure $\mathcal{X}=\left(X, \sqsubseteq, \oplus_{m \in M}, E_{m}\right)$ such that for each $m,\left(X, \sqsubseteq, \oplus_{m}, E_{m}\right)$ is a BI frame, and there is a preorder $\leq$ on $M$ satisfying:

$$
\text { (Operation Inclusion) } \quad m_{1} \leq m_{2} \rightarrow x \oplus_{m_{1}} y \subseteq x \oplus_{m_{2}} y .
$$

The (Operation Inclusion) condition together with the frame conditions of BI also imply an inclusion on unit sets:

LEMMA 3.4. Let $\mathcal{X}$ be an $M-B I$ frame. If $m_{1} \leq m_{2}$ then $E_{m_{2}} \subseteq E_{m_{1}}$.

Proof. Let $e_{2} \in E_{m_{2}}$. By (Unit Existence), there exists $e_{1} \in E_{m_{1}}$ such that $e_{2} \in e_{1} \oplus_{m_{1}} e_{2}$. By (Operation Inclusion), $e_{2} \in e_{1} \oplus_{m_{2}} e_{2}$, so (Unit Coherence) implies that $e_{1} \sqsubseteq e_{2}$, and then (Unit Closure) implies $e_{2} \in E_{m_{1}}$. So $E_{m_{2}} \subseteq E_{m_{1}}$.

To obtain a BI model over a given BI frame, we must provide a valuation, which defines which atomic propositions hold at each states in the frame. For the soundness of the proof system, it is important that the valuation is persistent: any formula true at a state remains true at any larger state. Formally, we define $M$-BI models as follows.

Definition 3.5 (Valuation and model). A persistent valuation is a map $\mathcal{V}: \mathcal{A P} \rightarrow \mathcal{P}(X)$ such that, for all $P \in \mathcal{A P}$, if $x \in \mathcal{V}(P)$ and $x \sqsubseteq y$ then $y \in \mathcal{V}(P)$. An $M$-BI model $(X, \mathcal{V})$ is an $M$-BI frame $\mathcal{X}=\left(X, \sqsubseteq, \oplus_{m}, E_{m}\right)$ associated with a persistent valuation $\mathcal{V}$ on it.

Next, we define which $M$-BI formula are true at a state in a $M$-BI model.

Definition 3.6. On model $(\mathcal{X}, \mathcal{V})$, we define the satisfaction relation $=_{\mathcal{V}}$ between states in $\mathcal{X}$ and $M$-BI formula: for $x \in \mathcal{X}$

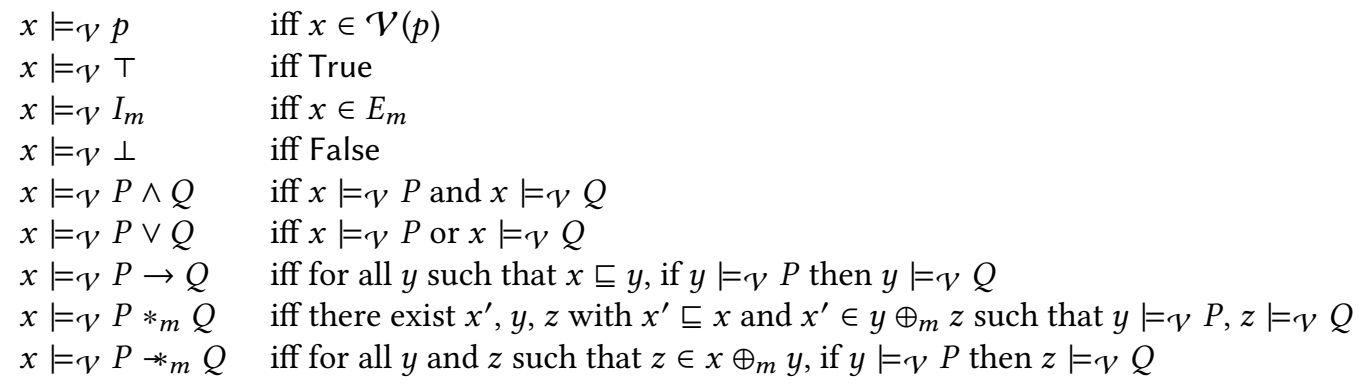

We say that a formula $P$ is valid in the model $(X, \mathcal{V})$, written as $\left.\mathcal{X}\right|_{\mathcal{V}} P$, iff $\left.x\right|_{\mathcal{V}} P$ for all $x \in \mathcal{X}$. We also say that $P$ is valid if and only if $P$ is valid in all models and write that as $=P$. Finally, we write $P=Q$ if and only if for any model $(X, \mathcal{V}), X=_{\mathcal{V}} P$ implies $\left.X\right|_{\mathcal{V}} Q$.

We prove the following theorem in the extended version.

Theorem 3.7. Let $P$ and $Q$ be any two $M-B I$ formulas. Then $P \models Q$ iff $P \vdash Q$.

The reverse direction of (soundness) is straightforward by induction on the proof derivation, but the forward direction (completeness) is less obvious; we use the duality-theoretic framework proposed by Docherty [2019] to establish this theorem. 


\subsection{Potential Models}

Our design of $M-\mathrm{BI}$ is mainly motivated by our intended model of negative association and probabilistic independence, which we will see in the next section, but the logic $M$-BI is quite flexible and we can see other natural models. Here we outline three models, inspired by the heap model of separation logic [Reynolds 2001].

Hierarchical heaps. In the heap model of separation logic, heaps are partial maps $h: \mathbb{N} \rightarrow$ Val from integer addresses to values, and heap combination ${ }^{{ }_{\text {heap }}}$ is a partial binary operation that takes the union if the two heaps have disjoint domains, and is not defined otherwise. In many systems, memory addresses are partitioned into larger units, for instance pages. We can define another partial binary operation $\circ_{\text {page }}$ that takes the union if the two heaps have disjoint domains and are defined on disjoint pages. Then, $h_{1} \circ_{\text {page }} h_{2} \subseteq h_{1}{ }^{\circ}$ heap $h_{2}$, so we can build a model of $M$-BI on the two-point pre-order. The two separating conjunctions $*_{\text {heap }}$ and $*_{\text {page }}$ then describe heap and page separation respectively, which could be useful for reasoning about which memory accesses may require a page-table lookup.

Strong separation logic. Heaps in separation logic can store values, but also addresses of other locations. In the standard heap model, two separate heaps must have disjoint domains but may store common addresses, i.e., they may hold dangling pointers to the same locations. Searching for a separation logic with better decidability properties, Pagel and Zuleger [2021] proposed a notion of strong separation logic, where two strongly-separated heaps can only hold common addresses that are already stored in stack variables. The resulting form of separation can be modeled by a separating conjunction $*_{s t}$, and the standard (weak) form of separation can be modeled by a separating conjunction $*_{w k}$. Since strong separation implies weak separation, we can again build a model of $M$-BI supporting both conjunctions on the two-point pre-order.

Tagged memory. In some security-focused architectures, pointers contain an address as well as a tag, indicating capabilities that may be performed with that piece of memory. To reason about these machines, we can consider a resource frame where states are pairs of $(h, p)$, where $h$ is a heap and $p$ is a permission (say shared access, or exclusive access). We can then consider four kinds of separation taking all combinations of heaps aliasing/non-

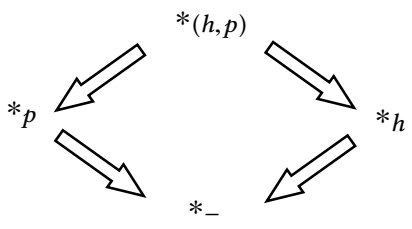

Fig. 2. The pre-order for $* m$ aliasing, and permissions compatible/incompatible. The result is a $M$-BI frame, with the lattice of separating conjunctions depicted in Figure 2. Assertions in models on this frame can reason about all four kinds of separation, where $*_{-}$degenerates to the standard conjunction $\wedge$.

\section{A MODEL OF NEGATIVE ASSOCIATION AND INDEPENDENCE}

In this section, we will present a $M$-BI model for reasoning about both probabilistic independence and negative association. Barthe et al. [2020] proposed a BI model that captures probabilistic independence, developed a program logic (PSL) to reason about independence in probabilistic programs. We will construct a BI model for negative association and combine it with the PSL model to obtain a 2-BI model for both probabilistic independence and negative association, where $2=\{1,2\}$ is the two-point set with pre-order $1 \leq 2$.

One may wonder if it is a simple exercise to replace the independence semantics of the separating conjunction in PSL by a semantics that capture negative association, but there are technical challenges. As we will show in Section 4.2, two intuitive BI model definitions fail to satisfy all frame conditions. To overcome the difficulties, in Section 4.3 we define a new notion of negative 
association that can express negative dependence of various strengths, and then define a model based on our new notion. All omitted proofs can be found in the extended version.

\subsection{Preliminaries and the PSL Model}

We need to introduce some notation to define the models.

First, we represent program states as memories. Let the set of all program variables be Var, and the set of all possible values be Val. For any finite set of variables $S \subseteq$ Var, a memory on $S$ is a map $S \rightarrow \mathrm{Val}$, and $\operatorname{Mem}[S]$ denotes the set of memories on $S$. For disjoint sets of variables $S, T \subseteq$ Var, and $m_{1} \in \operatorname{Mem}[S], m_{2} \in \operatorname{Mem}[T]$, we define $m_{1} \bowtie m_{2}$ to be the union of $m_{1}, m_{2}$.

Now we will introduce probabilistic memories. For a real-valued function $f$, we say that $x$ is in the support of $f$ if $f(x) \neq 0$. A countable distribution on a set $X$ is a countable support function $\mu: X \rightarrow[0,1]$ such that $\sum_{x \in X} \mu(x)=1$. Let $\mathcal{D}(X)$ denote the set of countable distributions on $X$. A probabilistic memory on variables $S$ is a distribution over Mem[S], so the set of probabilistic memories on $S$ is $\mathcal{D}(\operatorname{Mem}[S])$.

Next, we will need some constructions on distributions. A family of special distributions in $\mathcal{D}(\operatorname{Mem}[S])$ is Dirac distributions: for any $x \in \operatorname{Mem}[S]$, the Dirac distribution $\delta(x)$ puts all the weight on $x$, that is, for any $y \in \operatorname{Mem}[S], \delta(x)(y)=1$ if $x=y$, and $\delta(x)(y)=0$ otherwise. For any sets of variables $S \subseteq S^{\prime}$, we define the projection map $\pi_{S^{\prime}, S}$ to map a distribution $\mu$ on $\operatorname{Mem}\left[S^{\prime}\right]$ to a distribution on $\operatorname{Mem}[S]$ : for any $x \in \operatorname{Mem}[S]$,

$$
\pi_{S^{\prime}, S} \mu(x):=\sum_{x^{\prime} \in \operatorname{Mem}\left[S^{\prime}\right] \text { and } \mathbf{p}_{S}\left(x^{\prime}\right)=x} \mu\left(x^{\prime}\right),
$$

where $\mathbf{p}_{S}\left(x^{\prime}\right)$ is $x^{\prime}$ restricted on $S$. Often $S^{\prime}$ is clear, so we just write $\pi_{S}$ for $\pi_{S^{\prime}, S}$. Then, we can formally define independence of variables in a distribution:

Definition 4.1 (Independence). For any $\mu \in \mathcal{D}(\operatorname{Mem}[S])$, and disjoint $T_{1}, T_{2} \subseteq S$, we say $T_{1}, T_{2}$ are independent in $\mu$ if for any $x \in \operatorname{Mem}\left[T_{1} \cup T_{2}\right]$,

$$
\pi_{T_{1} \cup T_{2}} \mu(x)=\pi_{T_{1}} \mu\left(\mathbf{p}_{T_{1}}(x)\right) \cdot \pi_{T_{2}} \mu\left(\mathbf{p}_{T_{2}}(x)\right) .
$$

We then define the independent product $\otimes$ as: for any $\mu_{1} \in \mathcal{D}(\operatorname{Mem}[S]), \mu_{2} \in \mathcal{D}(\operatorname{Mem}[T])$,

$$
\mu_{1} \otimes \mu_{2}= \begin{cases}\emptyset & \text { if } S, T \text { not disjoint } \\ \left\{\mu \mid \text { for any } x \in \operatorname{Mem}[S \cup T], \mu(x)=\mu_{1}\left(\mathbf{p}_{S}(x)\right) \cdot \mu_{2}\left(\mathbf{p}_{T}(x)\right)\right\} & \text { if } S, T \text { disjoint }\end{cases}
$$

For any distribution $\mu \in \mathcal{D}(\operatorname{Mem}[S])$, we call $S$ the domain of $\mu$, denoted $\operatorname{dom}(\mu)$. By construction, if $\mu \in \mu_{1} \otimes \mu_{2}$, then $\operatorname{dom}\left(\mu_{1}\right)$ and $\operatorname{dom}\left(\mu_{2}\right)$ are independent in $\mu$, and $\mu$ is the unique element in $\mu_{1} \otimes \mu_{2}$. Simple calculations also show that if $\mu \in \mu_{1} \otimes \mu_{2}$, then $\pi_{S} \mu=\mu_{1}, \pi_{T} \mu=\mu_{2}$.

We can then present the Independence frame from Barthe et al. [2020] as the following BI frame. For simplicity, we restrict its states to probabilistic memories for now. ${ }^{2}$

Definition 4.2. Let $X=\cup_{S \subseteq \operatorname{Var}} \mathcal{D}(\operatorname{Mem}[S])$. Say $\mu \sqsubseteq \mu^{\prime}$ iff $\operatorname{dom}(\mu) \subseteq \operatorname{dom}\left(\mu^{\prime}\right)$ and $\pi_{\operatorname{dom}(\mu)} \mu^{\prime}=\mu$. Let $E_{\text {indep }}=X$. We call $\mathcal{X}_{\text {indep }}=\left(X, \sqsubseteq, \otimes, E_{\text {indep }}\right)$ the Independence structure.

This Independence structure $\mathcal{X}_{\text {indep }}$ is a BI frame.

\footnotetext{
${ }^{2}$ Technically we take a slightly different notion of BI frames that is more suitable for our purposes. Barthe et al. [2020] presents BI frames with partial, pre-ordered commutative monoids, which require a unique unit for all states. But we can encode their frame as our BI frame by taking its partial operation as an operation that returns sets of size at most one and defining the unit set $E$ to include their unique unit and be closed under $\sqsubseteq$.
} 


\subsection{Initial Attempts at a NA Model}

Our goal is to design a BI model $\mathcal{X}_{\mathrm{PNA}}$ that can capture negative association and can be combined with $\mathcal{X}_{\text {indep }}$. To be compatible with $\mathcal{X}_{\text {indep }}$, we let $\mathcal{X}_{\mathrm{PNA}}$ have the same set of states and the same pre-order as $\mathcal{X}_{\text {indep. }}$. The important remaining piece of the puzzle is the binary operation $\oplus$, which must satisfy the frame conditions.

One first attempt is to let $\mu_{1} \oplus \mu_{2}$ return the set of distributions that agree with $\mu_{1}, \mu_{2}$, and satisfy strong $N A$-we say $\mu$ satisfies strong NA if $\operatorname{dom}(\mu)$ satisfies NA.

Definition 4.3. (Attempt 1: Strong NA model) Let $X=\cup_{S \subseteq \operatorname{Var}} \mathcal{D}(\operatorname{Mem}[S])$. For $\mu, \mu^{\prime} \in X$, say $\mu \sqsubseteq \mu^{\prime}$ iff $\operatorname{dom}(\mu) \subseteq \operatorname{dom}\left(\mu^{\prime}\right)$ and $\pi_{\operatorname{dom}(\mu)} \mu^{\prime}=\mu$. Let $E_{s}=X$. Define $\oplus_{s}: X \times X \rightarrow \mathcal{P}(X)$ :

$\mu_{1} \oplus_{S} \mu_{2}=\left\{\mu \in \mathcal{D}(\operatorname{Mem}[S \cup T]) \mid \mu\right.$ satisfies strong NA, $\left.\pi_{S} \mu=\mu_{1}, \pi_{T} \mu=\mu_{2}, S \cap T=\emptyset\right\}$.

We call $\mathcal{X}_{s}=\left(X, \sqsubseteq, \oplus_{s}, E_{s}\right)$ the strong $N A$ structure.

Unfortunately, the strong NA structure fails to have the (Unit Existence) property: if $\mu$ does not satisfy strong NA, then there exists no $\mu^{\prime}$ that marginalizes to $\mu$ and satisfies strong NA, and thus no $e$ such that $\mu \in e \oplus_{s} \mu$. The failure of this property implies that whether or not two states can be combined depends on properties of the single states in isolation (e.g., whether a distribution satisfies strong NA), and not just on how the two states relate to each other; this is hard to justify if we are to read $\oplus$ as describing which pairs of states can be safely combined.

Looking for a different way of capturing NA, we can take inspiration from the $\mathcal{X}_{\text {indep }}$. There, $\mu_{1} \otimes \mu_{2}$ returns a distribution that agrees with $\mu_{1}, \mu_{2}$ and on which $\operatorname{dom}\left(\mu_{1}\right)$ are independent from $\operatorname{dom}\left(\mu_{2}\right)$. Thus, we can try letting $\mu_{1} \oplus \mu_{2}$ return distributions that agree with $\mu_{1}, \mu_{2}$ where any variable $x$ in $\operatorname{dom}\left(\mu_{1}\right)$ must be negatively associated with any variable $y$ in $\operatorname{dom}\left(\mu_{2}\right)$, but variables within $\operatorname{dom}\left(\mu_{1}\right)$ and variables within $\operatorname{dom}\left(\mu_{2}\right)$ need not be negatively associated. We call this notion weak NA.

Definition 4.4 (Weak NA). Let $S \subseteq$ Var be a set of variables, and let $A, B$ be two disjoint subsets of $S$. A distribution $\mu \in \mathcal{D}(\operatorname{Mem}[S])$ satisfies $(A, B)-N A$ if for every pair of both monotone or both antitone functions $f: \operatorname{Mem}[A] \rightarrow \mathbb{R}, g: \operatorname{Mem}[B] \rightarrow \mathbb{R}$, where we take the point-wise orders on $\operatorname{Mem}[A]$ and $\operatorname{Mem}[B]$, such that $f, g$ is either lower bounded or upper bounded, we have

$$
\mathbb{E}_{m \sim \mu}\left[f\left(\mathbf{p}_{A} m\right) \cdot g\left(\mathbf{p}_{B} m\right)\right] \leq \mathbb{E}_{m \sim \mu}\left[f\left(\mathbf{p}_{A} m\right)\right] \cdot \mathbb{E}_{m \sim \mu}\left[g\left(\mathbf{p}_{B} m\right)\right] .
$$

By definition, being $(A, B)$-NA for all disjoint $A, B \subseteq S$ is equivalent to strong NA on $S$. Now, we can try defining another model based on weak NA.

Definition 4.5. (Attempt 2: Weak NA model) Let $X=\cup_{S \subseteq \operatorname{Var}} \mathcal{D}(\operatorname{Mem}[S])$. For $\mu, \mu^{\prime} \in X, \mu \sqsubseteq \mu^{\prime}$ iff $\operatorname{dom}(\mu) \subseteq \operatorname{dom}\left(\mu^{\prime}\right)$ and $\pi_{\operatorname{dom}(\mu)} \mu^{\prime}=\mu$. Let $E_{w}=X$. Define $\oplus_{w}: X \times X \rightarrow \mathcal{P}(X)$ :

$\mu_{1} \oplus_{w} \mu_{2}=\left\{\mu \in \mathcal{D}(\operatorname{Mem}[S \cup T]) \mid \mu\right.$ satisfies $(S, T)$-NA, $\left.\pi_{S} \mu=\mu_{1}, \pi_{T} \mu=\mu_{2}, S \cap T=\emptyset\right\}$.

We call $\mathcal{X}_{w}=\left(X, \sqsubseteq, \oplus_{w}, E_{w}\right)$ the weak NA structure.

This weak NA structure satisfies most BI frame conditions, except that (Associativity) is unclear. In short, the definition of $\oplus_{w}$ and (Associativity) requires that: if $w$ satisfies $(R \cup S, T)$-NA and $(R, S)$ $\mathrm{NA}$, then $w$ also satisfies $(S, T)$-NA and $(R, S \cup T)$-NA. Now $w$ satisfies $(S, T)$-NA by projection closure, but it is unclear whether $w$ must satisfy $(R, S \cup T)$-NA; we leave this question as an interesting open problem. Failing to satisfy (Associativity) would lead to a logic where separating conjunction is not associative, and significantly more difficult to use. Since it is unknown whether $\mathcal{X}_{w}$ is a BI frame, we will define another structure to capture negative association. 


\subsection{Our NA Model}

Facing the problems with the strong NA structure and the weak NA structures, we will define a BI model for negative association based on a new notion of negative association called partition negative association (PNA). This notion interpolates weak NA and strong NA, in the following sense: $\{A, B\}$-PNA is equivalent to $(A, B)$-NA for disjoint $A, B \subseteq S$, and $\{\{s\} \mid s \in S\}$-PNA is equivalent to strong NA for distributions in $\mathcal{D}(\operatorname{Mem}[S])$.

Definition 4.6 (Partition Negative Association). We say a partition $\mathcal{S}^{\prime}$ coarsens a partition $\mathcal{S}$ if $\cup \mathcal{S}=\cup \mathcal{S}^{\prime}$ and for any $s^{\prime} \in \mathcal{S}^{\prime}, s^{\prime}=\cup \mathcal{R}$ for some $\mathcal{R} \subseteq \mathcal{S}$.

A distribution $\mu$ is $\mathcal{S}$-PNA if and only if for any $\mathcal{T}$ that coarsens $\mathcal{S}$, for any family of non-negative monotone functions (or family of non-negative antitone functions), $\left\{f_{A}: \operatorname{Mem}[A] \rightarrow \mathbb{R}^{+}\right\}_{A \in \mathcal{T}}$, where we take the point-wise order on $\operatorname{Mem}[A]$ for each $A \in \mathcal{T}$, we have

$$
\mathbb{E}_{m \sim \mu}\left[\prod_{A \in \mathcal{T}} f_{A}\left(\mathbf{p}_{A} m\right)\right] \leq \prod_{A \in \mathcal{T}} \mathbb{E}_{m \sim \mu}\left[f_{A}\left(\mathbf{p}_{A} m\right)\right] .
$$

We can use PNA to prove NA:

Theorem 4.7. Given a set of variables $S, S$ satisfies $N A$ in $\mu$ iff $\mu$ satisfies $\mathcal{S}$-PNA for any $\mathcal{S}$ partitioning $S$ iff $\mu$ satisfies $\{\{s\} \mid s \in S\}-P N A{ }^{4}$

We require PNA to be closed under coarsening, which helps us to prove the next structure we define is a BI frame.

Definition 4.8. Let $X_{\mathrm{PNA}}=\left(X, \sqsubseteq, \oplus, E_{\mathrm{PNA}}\right)$, where $X=E_{\mathrm{PNA}}=\cup_{S \subseteq \operatorname{Var}} \mathcal{D}(\operatorname{Mem}[S])$. For $\mu, \mu^{\prime} \in X$, say $\mu \sqsubseteq \mu^{\prime}$ iff $\operatorname{dom}(\mu) \subseteq \operatorname{dom}\left(\mu^{\prime}\right)$ and $\pi_{\operatorname{dom}(\mu)} \mu^{\prime}=\mu$. Define the operation $\oplus: X \times X \rightarrow \mathcal{P}(X)$ :

$\mu_{1} \oplus \mu_{2}=\left\{\mu \in \mathcal{D}(\operatorname{Mem}[S \cup T]) \mid \pi_{S} \mu=\mu_{1}, \pi_{T} \mu=\mu_{2}\right.$,

$\mu$ is $(\mathcal{S} \cup \mathcal{T})$-PNA for any partition $\mathcal{S}, \mathcal{T}$ such that

$\mu_{1}$ is $\mathcal{S}$-PNA, and $\mu_{2}$ is $\mathcal{T}$-PNA, and $(\cup \mathcal{S}) \cap(\cup \mathcal{T})=\emptyset$. $\}$

This definition of $\oplus$ interpolates $\oplus_{w}$ and $\oplus_{s}$, in the following sense.

Theorem 4.9. For any two states $\mu_{1}, \mu_{2} \in X, \mu_{1} \oplus_{s} \mu_{2} \subseteq \mu_{1} \oplus \mu_{2} \subseteq \mu_{1} \oplus_{w} \mu_{2}$.

The first inclusion is because $\mu$ satisfying strong NA implies $\mu$ is $\mathcal{R}$-PNA for any partition $\mathcal{R}$ on $\operatorname{dom}(\mu)$. The second inclusion is because $\mu_{1} \in \mathcal{D}(\operatorname{Mem}[S])$ satisfies $\{S\}$-PNA and $\mu_{2} \in \mathcal{D}(\operatorname{Mem}[T])$ satisfies $\{T\}$-PNA trivially, which implies any $\mu \in \mu_{1} \oplus \mu_{2}$ would satisfy $(S, T)$-NA.

Note that $\oplus$ is non-deterministic, and not just partial.

Theorem 4.10. There are distributions $\mu_{1}$, $\mu_{2}$ such that $\left|\mu_{1} \oplus \mu_{2}\right| \geq 2$.

Proof. Let $\mu_{1} \in \mathcal{D}(\operatorname{Mem}[\{x\}])$ and $\mu_{2} \in \mathcal{D}(\operatorname{Mem}[\{y\}])$ be uniform distribution over memories over $0 / 1$ variables $x, y$. Then the independent product $\mu_{\otimes} \in \mu_{1} \otimes \mu_{2}$ is in $\mu_{1} \oplus \mu_{2}$, because the projections to $x$ and to $y$ are $\mu_{1}$ and $\mu_{2}$ respectively, and $\mu_{\otimes}$ satisfies PNA since independence implies PNA (we will see this shortly in Theorem 4.12). But the one-hot uniform distribution $\mu_{o h}$ over variables $x$ and $y$, i.e., $\mu_{o h}([x \mapsto 1, y \mapsto 0])=\mu_{o h}([x \mapsto 0, y \mapsto 1])=1 / 2$, is also in $\mu_{1} \oplus \mu_{2}$, since again the projections match $\mu_{1}$ and $\mu_{2}$ and the one-hot distribution satisfies NA, and hence PNA. Since $\mu_{o h} \neq \mu_{\oplus}$, we are done.

\footnotetext{
${ }^{3}$ We restrict the family of functions to be non-negative: prior work like Joag-Dev and Proschan [1983] has assumed nonnegativity when working with notions of NA on partitions; furthermore, without that requirement, for partitions with odd number of components, PNA would be equivalent to independence, a strange property.

${ }^{4}$ Technically, we slightly modify Dubhashi and Ranjan [1998]'s NA when defining it in Definition 2.1 by in addition assuming that $f, g$ are bounded from one side. We add that condition to have a cleaner version of this theorem and Theorem 5.3. All our other results and properties we state about NA in Section 1 hold with or without this condition.
} 
Thus, we can build a BI frame on probabilistic memories, crucially using a non-deterministic combination operation on states [Docherty 2019].

THEOREM 4.11. The structure $X_{P N A}=\left(X, \sqsubseteq, \oplus, E_{P N A}\right)$ is a Down-Closed BI frame.

For the frame conditions where the previous attempts failed, (Unit Existence) holds by letting the unit $e$ to always be the trivial distribution on the empty set, and (Associativity) can be proved using the facts that PNA is closed under coarsening and coarsening commute with projections. We call $\mathcal{X}_{\mathrm{PNA}}$ the PNA model.

Now that we know the PNA frame is a BI frame and captures NA, we want to combine it with the PSL frame to construct a $M$-BI frame. To combine them, we need to show that for any $\mu_{1}, \mu_{2} \in X$,

$$
\mu_{1} \otimes \mu_{2} \subseteq \mu_{1} \oplus \mu_{2} \text {. }
$$

The inclusion is implied by the following theorem:

Theorem 4.12 (IndEPENDENCE IMPLies PNA). Let $S, T \subseteq$ Var be two disjoint sets of variables. Suppose $\mu_{1} \in \mathcal{D}(\operatorname{Mem}[S]), \mu_{2} \in \mathcal{D}(\operatorname{Mem}[T])$. If $\mu_{1}$ satisfies $\mathcal{S}$-PNA and $\mu_{2}$ satisfies $\mathcal{T}$-PNA, then any $\mu \in \mu_{S} \otimes \mu_{T}$ satisfies $\mathcal{S} \cup \mathcal{T}$-PNA.

This theorem generalizes the independence closure for NA from Theorem 2.3. Its proof, however, is more involved because PNA is more expressive and is closed under coarsening.

Thus, we can combine $\mathcal{X}_{\text {indep }}$ and $\mathcal{X}_{\mathrm{PNA}}$ into a 2-BI model.

Theorem 4.13. Let $2=\{1,2\}$ with pre-order $1 \leq 2$. Let $\oplus_{1}=\otimes, E_{1}=E_{\text {indep }}, \oplus_{2}=\oplus, E_{2}=E_{P N A}$. The structure $\mathcal{X}_{\mathcal{D}(\mathrm{Mem})}=\left(X, \sqsubseteq, \oplus_{1}, E_{1}, \oplus_{2}, E_{2}\right)$ is a 2-BI model.

Thus $\mathcal{X}_{\mathcal{D}(\mathrm{Mem})}$ is a $2-\mathrm{BI}$ frame on probabilistic memories.

\subsection{Combining with Deterministic Memory}

While we can model the program states of probabilistic programs as probabilistic memories, some variables might only get deterministic assignments. It is useful to know whether a variable is deterministic; for instance, a deterministic variable is automatically independent of other variables To keep track of deterministic variables, we want a 2-BI frame whose states distinguish deterministic memories and probabilistic memories. We will construct it using a general approach for composing $M$-BI models. In particular, we will compose $\mathcal{X}_{\mathcal{D}(\mathrm{Mem})}$ with a 2-BI frame on deterministic memories.

We can define the product of two $M$-BI frames if they share the same pre-order for indexing, $M$.

Definition 4.14. Let $M$ be a pre-order. Given two $M$-BI frames, $X_{1}=\left(X_{1}, \sqsubseteq_{1}, \oplus_{(1, m \in M)}, E_{(1, m \in M)}\right)$ and $\mathcal{X}_{2}=\left(X_{2}, \sqsubseteq_{2}, \oplus_{(2, m \in M)}, E_{(2, m \in M)}\right)$. The product frame, $\mathcal{X}=\mathcal{X}_{1} \times \mathcal{X}_{2}=\left(X, \sqsubseteq, \oplus_{m \in M}, E_{m \in M}\right)$ is defined as

- $X=X_{1} \times X_{2}$;

- $\left(x_{1}, x_{2}\right) \sqsubseteq\left(x_{1}^{\prime}, x_{2}^{\prime}\right)$ if and only if $x_{1} \sqsubseteq_{1} x_{1}^{\prime}$ and $x_{2} \sqsubseteq_{2} x_{2}^{\prime}$;

- For $m \in M,\left(x_{1}, x_{2}\right) \oplus_{m}\left(x_{1}^{\prime}, x_{2}^{\prime}\right)=\left\{\left(y_{1}, y_{2}\right) \mid y_{1} \in x_{1} \oplus_{1, m} x_{1}^{\prime} \wedge y_{2} \in x_{2} \oplus_{2, m} x_{2}^{\prime}\right\}$;

- $E_{m}=E_{1, m} \times E_{2, m}$.

Theorem 4.15. If $\mathcal{X}_{1}$ and $\mathcal{X}_{2}$ are two $M-B$ I frames, then $\mathcal{X}=\mathcal{X}_{1} \times \mathcal{X}_{2}$ is also an M-BI frame.

The proof is straightforward.

We now define a 2-BI frame modeling the independence and NA separation on the deterministic memories. Because deterministic variables are automatically independent of other variables, it is meaningless to check whether a set of deterministic variables can be separated into two disjoint subsets independent of each other. Thus, we do not require the separation of domain when modeling the independence and NA of deterministic variables: 
Definition 4.16. Let $X^{\prime}=\cup_{S \in \operatorname{Var}} \operatorname{Mem}[S]$, and $\sqsubseteq$ be $=$, and the unit set $E_{\mathrm{d}}=X^{\prime}$. Define $\oplus_{d}$ by:

$$
m_{1} \oplus_{d} m_{2}= \begin{cases}\left\{m_{1}\right\} & \text { if } m_{1}=m_{2} \\ \emptyset & \text { if } m_{1} \neq m_{2}\end{cases}
$$

Theorem 4.17. The structure $\mathcal{X}_{\mathrm{Mem}}=\left(X^{\prime}, \sqsubseteq_{d}, \oplus_{1}, E_{1}, \oplus_{2}, E_{2}\right)$, where $\oplus_{1}=\oplus_{2}=\oplus_{d}$ and $E_{1}=E_{2}=$ $E_{d}$, is a 2-BI frame.

Both $\mathcal{X}_{\mathrm{Mem}}$ and $\mathcal{X}_{\mathcal{D}(\mathrm{Mem})}$ are 2-BI frames, so we can take their product.

Corollary 4.18. $\mathcal{X}_{\text {comb }}=\mathcal{X}_{\mathrm{Mem}} \times \mathcal{X}_{\mathcal{D}(\mathrm{Mem})}$ is a 2-BI frame.

As desired, the states of $\mathcal{X}_{\text {comb }}$ describe both deterministic memories and probabilistic memories. Furthermore, restricting to the BI model in $X_{\text {comb }}$ with operators indexed by 1 recovers the probabilistic BI model in Barthe et al. [2020].

\section{PROGRAM LOGIC}

Given the model for NA developed in the previous section, we now have a suitable logic of assertions. In this section, we complete the picture by designing a program logic, named LINA, for reasoning about negative association and independence on probabilistic programs. We defer proofs and details to the extended version.

\subsection{Probabilistic Programs}

We consider probabilistic programs in a basic probabilistic imperative language PWHILE. Let $\mathcal{D V}, \mathcal{R} \mathcal{V}$ be disjoint countable subsets of Var that respectively contain all deterministic variables and all probabilistic variables. We consider program states to be a pair of a deterministic memory $\sigma$, and a distribution $\mu$ over the probabilistic memory, i.e., $(\sigma, \mu) \in \operatorname{Mem}[\mathcal{D} \mathcal{V}] \times \mathcal{D}(\operatorname{Mem}[\mathcal{R} \mathcal{V}])$.

Because we will want to decompose a program state as a product of two disjoint memories, each satisfying a sub-formula, we also want to interpret program expressions on memories whose probabilistic part is only on part of $\mathcal{R} \mathcal{V}$. These memories have type $\operatorname{Mem}[\mathcal{D V}] \times \mathcal{D}(\operatorname{Mem}[T])$ for some $T \subseteq \mathcal{R} \mathcal{V}$, and we call them configurations, denoted Config.

We assume all expressions in PWHILE are well-typed:

$$
\mathcal{E} \ni e::=\mathcal{D} \mathcal{V}|\mathcal{R} \mathcal{V}|[\mathcal{E}, \ldots, \mathcal{E}]|\mathcal{E}+\mathcal{E}| \mathcal{E} \wedge \mathcal{E} \mid \ldots
$$

Given an expression $e$, we can interpret it as $\operatorname{Mem}[\mathcal{D} \mathcal{V}] \times \operatorname{Mem}[T] \rightarrow$ Val for any $T \subseteq \mathcal{R} \mathcal{V}$ that includes all the free variables in $e$. We can also lift it to an interpretation from configurations to distributions of values, i.e., $\llbracket e \rrbracket: \operatorname{Mem}[\mathcal{D V}] \times \mathcal{D}(\operatorname{Mem}[T]) \rightarrow \mathcal{D}($ Val $)$.

We then define commands in PWHILE and again assume that they are well-typed:

$$
\begin{gathered}
C \ni c::=\operatorname{skip}|\mathcal{D V} \leftarrow \operatorname{Exp}| \mathcal{R} \mathcal{V} \leftarrow \operatorname{Exp}\left|\mathcal{R} \mathcal{V} \leftrightarrow \mathrm{U}_{T}\right| C ; C \\
\mid \text { if } \mathcal{E} \text { then } C \text { else } C \mid \text { while } \mathcal{E} \text { do } C .
\end{gathered}
$$

The randomization is introduced by the sampling command: $\mathcal{R} \mathcal{V} \$ \mathrm{U}_{T}$, where $\mathrm{U}_{T}$ stands for the uniform distribution on a multi-set $T$. We assume that the while loops terminate in finite steps on all inputs. We also assume that an expression assigned to a deterministic variable only mentions deterministic variables, and a command branching on a randomized expression does not assign to deterministic variables in its body/branches. This assumption ensures that deterministic variables will not receive randomized values during the execution. It is not difficult to enforce this condition by a syntactic restriction, which we omit for a cleaner presentation. 
Following the standard semantics for probabilistic programs due to Kozen [1981], we interpret $\mathrm{PWHILE}$ programs as transformers from program states to program states, i.e.,

$$
\llbracket c \rrbracket: \operatorname{Mem}[\mathcal{D} \mathcal{V}] \times \mathcal{D}(\operatorname{Mem}[\mathcal{R} \mathcal{V}]) \rightarrow \operatorname{Mem}[\mathcal{D} \mathcal{V}] \times \mathcal{D}(\operatorname{Mem}[\mathcal{R} \mathcal{V}])
$$

The semantics of PWHILE is standard.

In our examples, permutation distributions, uniform distributions over permutation $(A)$ :

Definition 5.1. Given a finite multi-set of $A$, a permutation of $A$ is a bijective function $\alpha: A \rightarrow A$. We let permutation $(A)$ be the multi-set of $A$ 's permutations. When $A$ has duplicates, we distinguish them using additional labels; so there are always $|A|$ ! elements in permutation $(A)$.

Let one-hot([n]) denote the set of length- $n$ one hot vectors. We then define the shorthands:

$$
\begin{aligned}
& \mathcal{R} \mathcal{V} \$ \operatorname{perm}(A) \triangleq \mathcal{R} \mathcal{V} \$ \mathrm{U}_{\text {permutation }(A)} \\
& \mathcal{R V} \$ o h(n) \triangleq \mathcal{R V} \& \mathrm{U}_{\text {one-hot }([n])} \\
& \text { if } b \text { then } c \triangleq \text { if } b \text { then } c \text { else skip. }
\end{aligned}
$$

\subsection{Assertion Logic: Atomic Propositions and Axioms}

Like other program logics, LINA has two layers: the program logic layer describing the relation between pre-conditions, programs and post-conditions, and the assertion logic layer describing program states. In Section 4, we have constructed a probabilistic model of 2-BI, $\mathcal{X}_{\text {comb }}$, whose states encompass all of Config, so our starting point for the assertion logic is this model. In this section, we introduce atomic propositions $\mathcal{A} \mathcal{P}$ for describing states in $\mathcal{X}_{\text {comb }}$ and some axioms that will hold on $\mathcal{X}_{\text {comb }}$.

We extend the core atomic formula from Barthe et al. [2020]. To talk about probabilities on program states distributions, we first define an event to be a function that maps a deterministic program configuration to 0 or 1 , and let $\mathcal{E} \mathcal{V}$ be a set of expressions that can be interpreted as event on deterministic configurations i.e., for any $e v \in \mathcal{E} \mathcal{V}$, $\llbracket e v \rrbracket: \operatorname{Mem}[\mathcal{D V}] \times \operatorname{Mem}[T] \rightarrow\{0,1\}$ for some $T \subseteq \mathcal{R} \mathcal{V}$. Since boolean expressions in the programming language can also be interpreted as this type, we will let $\mathcal{E} \mathcal{V}$ include all boolean expression. Let

$$
\mathcal{A P} \ni p::=\mathrm{U}_{T}\langle\mathcal{E}\rangle\left|\operatorname{Bern}_{p}\langle\mathcal{E}\rangle\right| \operatorname{Detm}\langle\mathcal{E}\rangle|\mathcal{E} \sim \mathcal{E}| \mathcal{E} \leq \mathcal{E}|\mathcal{E} \mathcal{V}=b| \operatorname{Pr}[\mathcal{E} \mathcal{V}] \bowtie \delta
$$

where $\bowtie \in\{=, \leq, \geq\}, b \in\{0,1\}$, and $\delta \in \mathbb{R}$ is a constant. In particular, for boolean expression $e$ and for $b \in\{0,1\}$, since we can also view $e$ as an event, $e \sim b$ and $e=b$ are both valid atomic propositions. We distinguish their notations $(\sim$ v.s. $=)$ because, in general, the left hand side of $\mathcal{E} \mathcal{V}=b$ may not be an expression and the left hand side of $\mathcal{E} \sim \mathcal{E}$ may not be an event.

We define the satisfaction of atomic proposition on program configurations as follows. Let FV $(e)$ be the set of free variables in expression $e$.

Definition 5.2 (Atomic Propositions). For $(\sigma, \mu) \in \mathcal{X}_{\text {comb }}$, define

- $(\sigma, \mu) \models \mathrm{U}_{T}\langle e\rangle$ iff $\mathrm{FV}(e) \subseteq \operatorname{dom}(\sigma) \cup \operatorname{dom}(\mu)$ and $\llbracket e \rrbracket(\sigma, \mu)$ is a distribution that assigns probability $\frac{1}{|T|}$ to each element of $T$;

- $(\sigma, \mu)=\operatorname{Bern}_{p}\langle e\rangle$ iff $\mathrm{FV}(e) \subseteq \operatorname{dom}(\sigma) \cup \operatorname{dom}(\mu)$ and $\llbracket e \rrbracket(\sigma, \mu)$ is a distribution that assign probability $p$ to 1 and probability $1-p$ to 0 , i.e., the Bernoulli distribution;

- $(\sigma, \mu) \vDash \operatorname{Detm}\langle e\rangle$ iff $\mathrm{FV}(e) \subseteq \operatorname{dom}(\sigma) \cup \operatorname{dom}(\mu)$ and $\llbracket e \rrbracket(\sigma, \mu)$ is a Dirac distribution;

- $(\sigma, \mu) \models e \sim e^{\prime}$ iff $\mathrm{FV}(e) \cup \mathrm{FV}\left(e^{\prime}\right) \subseteq \operatorname{dom}(\sigma) \cup \operatorname{dom}(\mu)$ and $\llbracket e \rrbracket(\sigma, m)=\llbracket e^{\prime} \rrbracket(\sigma, m)$ for any $m$ in the support of $\mu$;

- $(\sigma, \mu) \models e \leq e^{\prime}$ iff $\mathrm{FV}(e) \cup \mathrm{FV}\left(e^{\prime}\right) \subseteq \operatorname{dom}(\sigma) \cup \operatorname{dom}(\mu)$ and $\llbracket e \rrbracket(\sigma, m) \leq \llbracket e^{\prime} \rrbracket(\sigma, m)$ for any $m$ in the support of $\mu$;

- $(\sigma, \mu) \models e v=b$ if for any $m$ in the support of $\mu$, $e e v \rrbracket(\sigma, m)=b$. 
- $(\sigma, \mu) \mid=\operatorname{Pr}[e v] \bowtie \delta$ iff the probability of event $\llbracket e v \rrbracket$ in $(\sigma, \mu)$, defined to be $\operatorname{Pr}_{(\sigma, \mu)}[e v]=$ $\sum_{m \in \operatorname{Mem}[\operatorname{dom}(\mu)]} \mu(m) \cdot \llbracket e v \rrbracket(\sigma, m)$, satisfies $\operatorname{Pr}_{(\sigma, \mu)}[e] \bowtie \delta$.

We use the abbreviations:

- $\langle e\rangle \triangleq e \sim e$. That is, $(\sigma, \mu) \mid=\langle e\rangle$ holds if all of the variables in $e$ are defined in $\sigma$ and $\mu$.

- $\mathrm{OH}_{N}\langle e\rangle \triangleq \mathrm{U}_{\text {one-hot([N]) }}\langle e\rangle$.

- For multi-set $A, \operatorname{Perm}_{A}\langle e\rangle \triangleq \mathrm{U}_{\text {permutation(A) }}\langle e\rangle$.

For any operation $\odot \in\{\wedge, \vee, \circledast, *\}$, we pick the corresponding big-operation $\odot \in\{\wedge, \vee, \circledast, *\}$ to be their iterated version.

With the atomic propositions and abbreviations, we can formally state that $\mathcal{X}_{\text {comb }}$ captures NA.

THEOREM 5.3. Let $S$ be any subset of $\mathcal{R} \mathcal{V}$. A set of randomized program variables $Y=\left\{y_{i} \mid 0 \leq\right.$ $i<K\}$ satisfies $N A$ in distribution $\mu \in \mathcal{D}(\operatorname{Mem}[S])$ if and only if for any deterministic memory $\sigma \in \operatorname{Mem}[\mathcal{D} \mathcal{V}]$, we have $(\sigma, \mu)=\circledast_{i=0}^{K}\left\langle y_{i}\right\rangle$.

In the $\mathcal{X}_{\text {comb }}$ model, all axioms from Barthe et al. [2020, Lemma 3, 4] still hold, and we have new axioms for the negative association conjunction and the permutation distribution.

Lemma 5.4. Let $x_{\gamma}$ be variables. The following axioms are valid in $\mathcal{X}_{\text {comb }}$ :

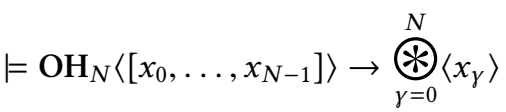

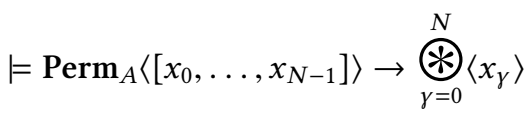

The two axioms follow from Theorem 2.2, which shows that random variables in one-hot distributions and permutation distributions are NA, and Theorem 5.3, which shows that $\circledast$ captures the NA of random variables. We can also encode the monotone map closure in Theorem 2.3 as an axiom in the logic.

Lemma 5.5 (Monotone map). Let $x, x_{\gamma, \alpha}$ and $y_{\gamma}$ be variables. The following is valid in $\mathcal{X}_{\text {comb: }}$ :

$$
\vDash \bigoplus_{\gamma=0}^{N}\left(\bigwedge_{\alpha=0}^{K_{\gamma}+1}\left\langle x_{\gamma, \alpha}\right\rangle\right) \wedge \bigwedge_{\gamma=0}^{N} y_{\gamma}=f_{\gamma}\left(x_{\gamma, 0}, \ldots, x_{\gamma}, K_{\gamma}\right) \rightarrow \bigoplus_{\gamma=0}^{N}\left\langle y_{\gamma}\right\rangle
$$

$$
\text { when } f_{1}, \ldots, f_{N} \text { all monotone or all antitone }
$$

(Mono-Map)

When we establish NA from permutation distributions, it is preserved under not only monotone/antitone maps but also any element-wise homogeneous maps. The reason is that fixing a multi-set and a permutation, permuting first and then applying the same map on each element is the equivalent to applying the map on each element and then permuting. So applying homogeneous maps on a permutation distribution gives another permutation distribution. We can capture this property in an axiom.

Lemma 5.6 (Permutation MAP). Let $x_{\gamma}$ be variables, and $f(A)$ be $\{f(a) \mid a \in A\}$. The following axiom is valid in $\mathcal{X}_{\text {comb }}$ :

$$
=\operatorname{Perm}_{A}\left\langle\left[x_{1}, \ldots, x_{N}\right]\right\rangle \wedge y \sim\left[f\left(x_{1}\right), \ldots, f\left(x_{N}\right)\right] \rightarrow \operatorname{Perm}_{f(A)}\langle y\rangle
$$




\subsection{Restricting the Assertion Language}

When designing a separation logic for reasoning about negative association and independence, we sometimes want to separate out a smaller configuration $\left(\sigma^{\prime}, \mu^{\prime}\right)$ inside a given program state $(\sigma, \mu) \vDash \phi$, such that $\left(\sigma^{\prime}, \mu^{\prime}\right)$ satisfies some sub-formula of $\phi$. In the program logic we will present in Section 5.4, the soundness of RCASE, Const, Frame and NegFrame rules all rely on the ability to do that. To ensure there exists such a smaller configuration, we require the assertion logic to satisfy a key condition called restriction, which says that to check whether a configuration satisfies $\phi$, it suffices to check whether the configuration's projection on $\operatorname{FV}(\phi)$ satisfies $\phi$. We identify a subset of $M$-BI formulas that satisfy the restriction property when interpreted on states in $\mathcal{X}_{\text {comb: }}$ :

Definition 5.7. We define $M-\mathrm{BI}_{\text {restricted }}$ as

$$
M-\mathrm{BI}_{\text {restricted }} \ni P, Q::=p \in \mathcal{A P}|\top| \perp|P \wedge Q| P \vee Q|P \rightarrow Q| P * Q|P * Q| P \circledast Q
$$

where $\mathcal{A P}$ is defined as in Equation (1).

$M-\mathrm{BI}_{\text {restricted }}$ omits multiplicative identities $I_{m}$ because on $\mathcal{X}_{\text {comb }}$ they are all equivalent to $\mathrm{T}$. The only limitation is that $M-\mathrm{BI}_{\text {restricted }}$ excludes the use of $-\circledast$.

Theorem 5.8 (Restriction). Let $(\sigma, \mu)$ be any configuration, and let $\phi$ be an $M-B I_{\text {restricted }}$ formula interpreted on $\mathcal{X}_{\text {comb }}$, Then, for any $m \in \operatorname{Mem}[\mathcal{D} \mathcal{V} \backslash F V(\phi)]$,

$$
(\sigma, \mu) \models \phi \Longleftrightarrow\left(\mathbf{p}_{F V(\phi)} \sigma \bowtie m, \pi_{F V(\phi)} \mu\right)=\phi .
$$

Indeed, we can exhibit a counterexample showing that $-\circledast$ does not satisfy restriction.

Theorem 5.9. There exists $(\sigma, \mu) \in$ Config and formula $\phi$ such that $(\sigma, \mu) \models \phi$ but $\left(\sigma, \pi_{F V(\phi)}\right) \not k$.

In the following, we will consider $M-\mathrm{BI}_{\text {restricted }}$ formula on the $\mathcal{X}_{\text {comb }}$ model as the assertion logic.

\subsection{The Program Logic}

We now introduce the program logic layer of LINA. Judgements in LINA have the form $\{P\} c\{Q\}$, where $c \in C$ is a probabilistic program, and $P, Q \in M-\mathrm{BI}_{\text {restricted }}$ are restricted assertions.

Definition 5.10 (Validity). A LINA judgment is valid, written $=\{P\} c\{Q\}$, if for all $(\sigma, \mu) \in$ $\operatorname{Mem}[\mathcal{D} \mathcal{V}] \times \mathcal{D}(\operatorname{Mem}[\mathcal{R} \mathcal{V}])$ such that $(\sigma, \mu) \models P$, we have $\llbracket c \rrbracket(\sigma, \mu) \models Q$.

Next, we present the proof system of LINA. Since our assertions are a conservative extension of assertions from PSL, most of the rules carry over unchanged; we list existing rules in Figure 3. Here, we comment on the new and generalized rules, which we list in Figure 4.

NA frame rule. Our most important addition is the frame rule for the negative association conjunction $\circledast$. Informally, the NEGFrame rule says that if a set of variables $X$ is negatively associated with another set of variables $Y$ that satisfy $\eta$ in a program state, and the program $c$ performs a monotone operation $f$ on $X$ and stores the result in a variable $y$, then in the resulting program state, $y$ and the untouched variables $Y$ will also be negatively associated, and $Y$ will still satisfy $\eta$. Like the Frame rule for independence $*$, the NegFrame rule uses syntactic restrictions to control which variables the program may read and write. The three sets of variables $\mathrm{RV}(c), \mathrm{WV}(c), \operatorname{MV}(c)$ represent the variables that $c$ may read from, must write to, and may modify, respectively; these sets can be defined by induction on the syntax of the program. Roughly, the side conditions guarantee the program $c$ does not read from or modify $Y$, the set of variables satisfying $\eta$; they in addition guarantee that $X$, the domain of the monotone map will not be modified by $c$, and $y$, the codomain of the monotone map does not belong to $Y$. 


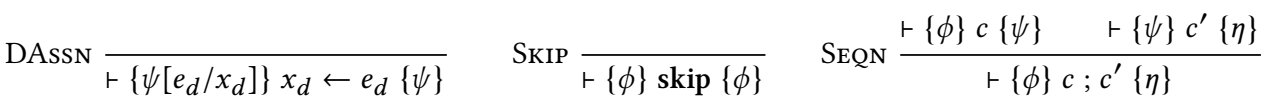

$$
\begin{aligned}
& \operatorname{CoND} \frac{\vdash\{\phi \wedge b \sim t t\} c\{\psi\} \quad \vdash\{\phi \wedge b \sim f f\} c^{\prime}\{\psi\} \quad \vDash \phi \rightarrow \operatorname{Detm}\langle b\rangle}{\vdash\{\phi\} \text { if } b \text { then } c \text { else } c^{\prime}\{\psi\}} \\
& \text { Loop } \frac{\vdash\{\phi \wedge b \sim t t\} c\{\phi\} \quad \vDash \phi \rightarrow \operatorname{Detm}\langle b\rangle}{\vdash\{\phi\} \text { while } b \operatorname{do} c\{\phi \wedge b \sim f f\}} \quad \text { RAssN } \frac{x_{r} \notin \mathrm{FV}\left(e_{r}\right)}{\vdash\{T\} x_{r} \leftarrow e_{r}\left\{x_{r} \sim e_{r}\right\}} \\
& \operatorname{RSAMP} \frac{x_{r} \notin \mathrm{FV}(\phi)}{\vdash\{T\} x_{r} \$ \mathrm{U}_{S}\left\{\mathrm{U}_{S}\left\langle x_{r}\right\rangle\right\}} \quad \operatorname{RSAMP}^{*} \frac{1 \phi\} x_{r} \notin \mathrm{U}_{S}\left\{\phi * \mathrm{U}_{S}\left\langle x_{r}\right\rangle\right\}}{\vdash\{1} \\
& \text { WeAK } \frac{\vdash\{\phi\} c\{\psi\} \quad=\phi^{\prime} \rightarrow \phi \wedge \psi \rightarrow \psi^{\prime}}{\vdash\left\{\phi^{\prime}\right\} c\left\{\psi^{\prime}\right\}} \quad \text { True } \frac{}{\vdash\{T\} c\{T\}} \\
& \operatorname{CoNJ} \frac{\vdash\left\{\phi_{1}\right\} c\left\{\psi_{1}\right\} \quad \vdash\left\{\phi_{2}\right\} c\left\{\psi_{2}\right\}}{\vdash\left\{\phi_{1} \wedge \phi_{2}\right\} c\left\{\psi_{1} \wedge \psi_{2}\right\}} \quad \operatorname{CASE} \frac{\vdash\left\{\phi_{1}\right\} c\left\{\psi_{1}\right\} \quad \vdash\left\{\phi_{2}\right\} c\left\{\psi_{2}\right\}}{\vdash\left\{\phi_{1} \vee \phi_{2}\right\} c\left\{\psi_{1} \vee \psi_{2}\right\}} \\
& \text { Const } \frac{\vdash\{\phi\} c\{\psi\} \quad \mathrm{FV}(\eta) \cap \mathrm{MV}(c)=\emptyset}{\vdash\{\phi \wedge \eta\} c\{\psi \wedge \eta\}} \\
& \operatorname{FrAmE} \frac{\vdash\{\phi\} c\{\psi\} \quad \mathrm{FV}(\eta) \cap \mathrm{MV}(c)=\emptyset \quad \mathrm{FV}(\psi) \subseteq T \cup \operatorname{RV}(c) \cup \mathrm{WV}(c) \quad=\phi \rightarrow\langle T \cup \operatorname{RV}(c)\rangle}{\vdash\{\phi * \eta\} c\{\psi * \eta\}}
\end{aligned}
$$

Fig. 3. LINA rules: from PSL.

$$
\begin{aligned}
& \eta \in \mathrm{CC} \quad F_{\mathrm{Mem}} \eta \rightarrow \bigvee_{\alpha \in S} \eta_{\alpha} \quad \psi \in \mathrm{CM} \quad \forall \alpha \in S . \vdash\left\{\phi * \eta_{\alpha}\right\} c\{\psi\} \\
& \text { RCASE } \frac{\alpha \in S}{\vdash\{\phi * \eta\} c\{\psi\}} \\
& \vDash \phi \rightarrow\langle\mathrm{RV}(c)\rangle \quad \mathrm{FV}(\eta) \cap \mathrm{MV}(c)=\emptyset \quad X \subseteq \mathrm{RV}(c) \backslash \mathrm{MV}(c) \quad y \notin \mathrm{FV}(\eta) \\
& \text { NegFrame } \frac{\vdash\{\phi\} c\{y \sim f(X)\} \quad f \text { is a monotor }}{\vdash\{\phi \circledast \eta\} c\{\langle y\rangle \circledast \eta\}} \\
& \text { ProbBound } \frac{\vdash\left\{e v_{1}=1\right\} c\left\{\operatorname{Pr}\left[e v_{2}\right] \leq \delta\right\}}{\vdash\left\{\operatorname{Pr}\left[e v_{1}\right] \geq 1-\epsilon\right\} c\left\{\operatorname{Pr}\left[e v_{2}\right] \leq \delta+\epsilon\right\}}
\end{aligned}
$$

Fig. 4. LINA rules: new and extended.

Generalized random case analysis. As a more minor extension, we also generalize the randomized case analysis rule from PSL in RCASE. At a high level, this rule allows reasoning by case analysis on a property $\eta$ of the program memory (e.g., whether a variable is true or false). Since the input is a distribution, which may have some probability of $\eta$ holding, and some probability of $\eta$ not holding, soundness of the rule is a delicate matter requiring several technical side conditions. The original rule in PSL only allowed case analysis on a Boolean expression; we generalize this rule to 
allow a case analysis on any finite number of cases (e.g., performing case analysis on the value of a bounded variable).

To explain this rule, we first introduce the side conditions in order. We say that a formula $\eta$ is closed under conditioning (CC) if for any $(\sigma, \mu)=\eta$, for any $m$ in the support of $\mu,(\sigma, \delta(m)) \vDash \eta$. In the second condition, $\models_{\text {Mem }} \phi$ denotes that for any $\sigma \in \operatorname{Mem}[\mathcal{D} \mathcal{V}], m \in \operatorname{Mem}[T]$ where $T \subseteq \mathcal{R} \mathcal{V}$, $(\sigma, \delta(m))=\phi$, which says $\phi$ is valid on all effectively deterministic configurations. Finally, we say that a formula $\phi$ is closed under mixtures $(\mathrm{CM})$ if $\left(\sigma, \mu_{1}\right) \models \phi,\left(\sigma, \mu_{2}\right) \models \phi$ and $\mu$ is a convex combination of $\mu_{1}, \mu_{2}$ together imply $\left(\sigma, \mu^{\prime}\right)=\phi$.

Then, the rule RCASE says if an assertion $\eta$ is independent from the rest of the assertions in the pre-condition, $\eta$ is closed under conditioning, and the post-condition $\psi$ is closed under mixtures, then we can perform case analysis on $\eta$ to derive $\{\phi * \eta\} c\{\psi\}$. Intuitively, every memory $m$ in the support of the input memory distribution satisfies $\eta_{a}$ for some case $a \in S$. The main premise shows that the output distribution of program $c$ from any such input $m$ satisfies $\psi$. Then, since any distribution $\mu$ on inputs is a convex combination of such memories $m$, and $\psi$ holds on each conditional output distribution, we have $\psi$ holds on the entire output distribution by convex closure.

Bounding bad events. In addition, we present the rule ProBBound to facilitate bounding tail probabilities. It says that if the pre-condition $e v_{1}=1$ guarantees that event $e v_{2}$ happens for at most $\delta$ probability after command $c$, then in general, event $e v_{2}$ happens for at most probability $\delta+\epsilon$ after $c$, where $\epsilon$ upper bounds the probability that $e v_{1}$ is not true in the pre-condition. The validity of this rule uses the law of total probability, which says for any two events $e v_{1}$ and $e v_{2}$,

$$
\begin{aligned}
\operatorname{Pr}\left(e v_{1}\right) & =\operatorname{Pr}\left(e v_{1} \mid e v_{2}\right) \cdot \operatorname{Pr}\left(e v_{2}\right)+\operatorname{Pr}\left(e v_{1} \mid \neg e v_{2}\right) \cdot \operatorname{Pr}\left(\neg e v_{2}\right) \\
& \leq \operatorname{Pr}\left(e v_{1} \mid e v_{2}\right)+\operatorname{Pr}\left(\neg e v_{2}\right) .
\end{aligned}
$$

As expected, the LINA proof system is sound.

TheOrem 5.11. (Soundness of LINA) If $\vdash\{\phi\} c\{\psi\}$ is derivable, then it is valid: $=\{\phi\} c\{\psi\}$.

\section{EXAMPLES}

Now that we have introduced LINA, we present a series of formalized case studies. Our examples are extracted from various algorithms using hashing and balls-into-bins processes.

\subsection{Preliminaries: Probabilities, Expectations, Concentration bounds}

Our examples will use a handful of standard facts about probability distributions, encoded as axioms in the assertion logic. We will generally mention these axioms before they are used, but here we introduce one fact that we will use through all of our examples: the Chernoff bound.

In each of our examples we will establish negative dependence of a sequence of random variables $\left\{X_{i}\right\}_{i}$ and apply a concentration bound: a theorem showing that the sum $X_{1}+\cdots+X_{n}$ is usually close to its expected value. This kind of analysis is useful for establishing high-probability guarantees of randomized algorithms, e.g., showing that the error of a random estimate is at most 0.01 with probability at least $99 \%$.

Theorem 6.1 (Chernoff bound For NA variables [Dubhashi And Ranjan 1998]). Let $X_{1}, \ldots, X_{n}$ be a sequence of $N A$ random variables, each bounded in $[0,1]$, and let $Y=\sum_{i=1}^{n} X_{i}$. Then for any failure probability $\beta \in(0,1]$, we have:

$$
\operatorname{Pr}[|Y-\mathbb{E}[Y]| \geq T(\beta, n)] \leq \beta \quad \text { where } T(\beta, n)=\sqrt{(n / 2) \ln (2 / \beta)} .
$$

To hide complex numerical bounds, we use the notation $T(\beta, n)$ for the above function throughout. In our assertion logic, the Chernoff bound can be encoded as the following axiom schema: 


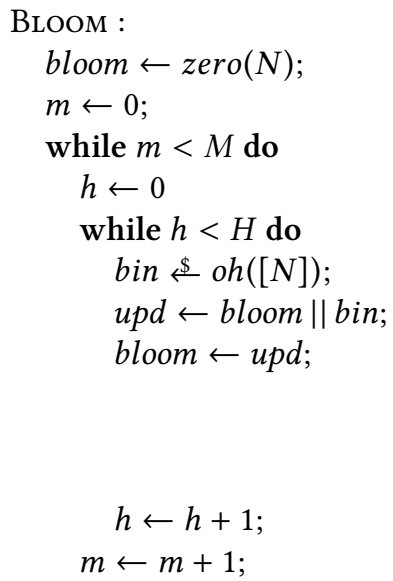

(a) Higher-level version

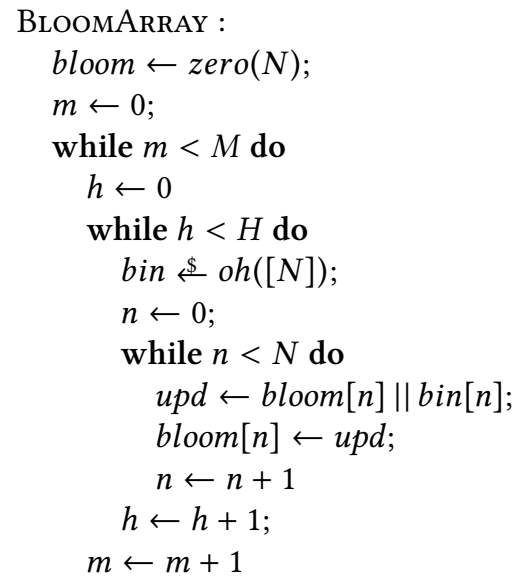

(b) Array version

Fig. 5. Bloom filter examples

TheOrem 6.2 (Chernoff Bound, AXIOM). Let $\left\{x_{\alpha}\right\}$ be a family of variables indexed by $\alpha$, where each variable is bounded in $[0,1]$ and is a monotone function of its program variables. Then for any $\beta \in(0,1]$, the following axiom schema is sound in our model:

$$
=\overbrace{\alpha=0}^{N}\left\langle x_{\alpha}\right\rangle \rightarrow \operatorname{Pr}\left[\left|\sum_{\alpha=0}^{N} x_{\alpha}-\mathbb{E}\left[\sum_{\alpha=0}^{N} x_{\alpha}\right]\right| \geq T(\beta, n)\right] \leq \beta
$$

We will also use a new expression in our assertions: $\mathbb{E}[f]$, where $f$ is a non-negative and bounded numeric expression, denotes the expected value of $f$ in the current program configuration. We also observe the following conventions throughout the examples: logical variables are denoted by Greek $(\alpha, \beta, \gamma, \ldots)$ and capital Roman letters $(M, N, K, \ldots)$. Program variables start with lower-case Roman letters $(x, y, z, \ldots)$.

\subsection{Bloom Filter, High-Level}

Next, we revisit the Bloom filter example introduced in Section 2. We show how to translate the informal argument in Section 2 into formal proofs in our program logic. First, we will analyze the process of adding items into a Bloom filter bloom and prove that the entries in bloom are negatively associated at the end of the process. Second, we will analyze a program that checks the membership of a new item in a given Bloom filter and show how to bound its false positive rate. Last, we combine them together into one proof that bounds the false positive rate of a Bloom filter with $M$ elements.

Proving NA of bloom. We reproduce the code for BLоOм in Figure 5a. This program is a higherlevel version of the program in Figure 5b, which performs array operations bit-by-bit. We align the two versions so that the equivalent operations are side-by-side. We will demonstrate our program logic on the higher-level version first and analyze the array version later in Section 6.3.

Recall that the code models inserting $M$ distinct elements into a Bloom filter backed by an array bloom of length $N$, where each element is hashed by $H$ functions, each producing an element of $[N]$ uniformly at random. We refer to the outer loop as outer, and the inner loop as inner. For both the outer and the inner loop, we apply the rule Loop with the loop invariant: $\circledast_{\beta=0}^{N}\langle$ bloom $[\beta]\rangle$. 
We consider the inner loop first. We show that the invariant is preserved by the body of inner. After the oh sampling command, RSAMP* gives:

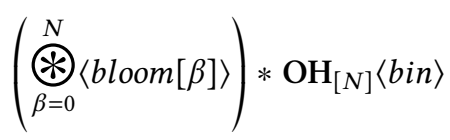

By negative association of the one-hot distribution (OH-PNA), we get

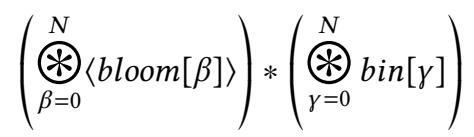

which implies

$$
(\overbrace{\beta=0}^{N}\langle\operatorname{bloom}[\beta]\rangle) \circledast(\overbrace{\gamma=0}^{N} \operatorname{bin}[\gamma])
$$

using WEAK. Rearranging terms, this is equivalent to

$$
\circledast_{\beta=0}^{N}\langle\operatorname{bloom}[\beta]\rangle \circledast\langle\operatorname{bin}[\beta]\rangle .
$$

After the assignment to upd, we have:

$$
\left(\circledast_{\beta=0}^{N}\langle\text { bloom }[\beta]\rangle \circledast\langle\text { bin }[\beta]\rangle\right) \wedge \text { upd } \sim \text { bloom } \| \text { bin. }
$$

Because || is monotone, applying the monotone mapping axiom (Mono-Map) gives us:

$$
\overbrace{\beta=0}^{N}\langle u p d[\beta]\rangle \text {. }
$$

Using the assignment rule (RAssN) on the assignment to bloom shows that the loop invariant is preserved by the inner loop. Thus, Loop gives:

$$
\{\overbrace{\beta=0}^{N}\langle\text { bloom }[\beta]\rangle\} \text { inner }\left\{\stackrel{\circledast}{*}_{\beta=0}^{N}\langle\text { bloom }[\beta]\rangle\right\}
$$

Next, we turn to the outer loop. The argument showing that the invariant is preserved by the outer loop follows by a straightforward argument, since the outer loop only modifies bloom through the inner loop, so Loop gives:

$$
\left\{\bigoplus_{\beta=0}^{N}\langle\text { bloom }[\beta]\rangle\right\} \text { outer }\left\{\bigoplus_{\beta=0}^{N}\langle\operatorname{bloom}[\beta]\rangle\right\}
$$

Then, we have:

$$
\{\top\} \operatorname{Bloom}\left\{\bigoplus_{\beta=0}^{N}\langle\text { bloom }[\beta]\rangle\right\}
$$

because initializing bloom to the all-zeros vector, a deterministic value, establishes the loop invariant. This judgment shows that the bloom vector satisfies NA at the end of the program. 
Bounding the false positive rate. Now, we turn to verifying a bound on the false positive rate of the Bloom filter. Recall that a false positive occurs if when querying with an element that was not inserted, the filter returns true. We can encode the membership check of a new element as a program Снескмем (H, bloom), listed in Figure 6, which hashes the new element into $H$ uniformly random positions and checks if these positions are all set to one in the filter. If so, the Bloom filter will report that the new element is in set, when it was never inserted-a false positive.

To verify the false positive rate, we place the program CheскMem $(H, b l o o m)$ immediately after Bloom, and then verify a bound on the probability that allhit is 1 at the end of the combined program. We first apply the Chernoff bound to the NA variables (NA-Chernoff) to prove that, with high probability, the number of occupied bins in BLOOM is near its mean with high probability:

$$
\{\top\} \text { Bцоом }\left\{\operatorname{Pr}\left[\mid \sum_{\beta=0}^{N} \text { bloom }[\beta]-\mathbb{E}\left[\sum_{\beta=0}^{N} \text { bloom }[\beta]\right] \mid \geq T(\delta, N)\right] \leq \delta\right\} .
$$

This concentration bound implies that a tail bound, which says with high probability $\sum_{\beta=0}^{N}$ bloom $[\beta]$ is upper bounded by its expected value plus $T(\delta, N)$,

$$
\{\top\} \text { B Lоom }\left\{\operatorname{Pr}\left[\sum_{\beta=0}^{N} \operatorname{bloom}[\beta]<\mathbb{E}\left[\sum_{\beta=0}^{N} \operatorname{bloom}[\beta]\right]+T(\delta, N)\right] \geq 1-\delta\right\} \text {. }
$$

Then we analyze СнескМем and show in the extended version that

$$
\left\{\sum_{\beta=0}^{N} \operatorname{bloom}[\beta]<K\right\} \text { СнескMем }\left\{\operatorname{Pr}[\text { allhit }] \leq(K / N)^{H}\right\} .
$$

Then, by the ProвBound rule and basic axioms about probabilities, we have

$$
\left\{\operatorname{Pr}\left[\sum_{\beta=0}^{N} \operatorname{bloom}[\beta]<K\right] \geq 1-\delta\right\} \text { CнескMEM }\left\{\operatorname{Pr}[\text { allhit }] \leq(K / N)^{H}+\delta\right\} .
$$

We then use SEQN to combine the proved judgements for Bloom (2) and СнескМем (3) to derive that, for any $\delta$,

$$
\{\top\} \text { Bloom; СнескMem }\left\{\operatorname{Pr}[\text { allhit }] \leq\left(\frac{\mathbb{E}\left[\sum_{\beta=0}^{N} \text { bloom }[\beta]\right]+T(\delta, N)}{N}\right)^{H}+\delta\right\} .
$$

Since allhit is 1 exactly when there is a false positive, this judgment proves an upper bound on the false positive rate of the Bloom filter. ${ }^{5}$

\footnotetext{
${ }^{5}$ The precise expected value is $N \cdot\left(1-(1-1 / N)^{M \cdot H}\right)$, a fact which can also be shown in our logic. Roughly speaking, this fact follows because each element of bloom is the logical-or of $M \cdot H$ probabilistically independent bits, each 1 with probability $1 / N$ and 0 otherwise. This argument does not rely on negative association.
} 


\subsection{Bloom Filter, Low-Level}

The previous Bloom filter uses a vector operation bloom $\|$ bin to transform an array of negatively associated values. We next consider a lower-level version of the previous example, BloomArRAY, in Figure 5b, where the vector operation is replaced by a loop that applies the Boolean-or.

Let outer and mid be the outer-most and second outer-most loops, and let inner be the inner-most loop. Again, our goal is to show that the vector bloom is negatively associated at the end of the program. We first prove the following judgment for inner:

$$
\left\{\bigoplus_{\beta=0}^{N}\langle\text { bloom }[\beta]\rangle * \circledast_{\gamma=0}^{N}\langle\text { bin }[\gamma]\rangle\right\} \text { inner }\left\{\bigoplus_{\beta=0}^{N}\langle\text { bloom }[\beta]\rangle \circledast \bigoplus_{\gamma=n}^{N}\langle\text { bin }[\gamma]\rangle\right\}
$$

We will apply the rule Loop on inner with the following loop invariant:

$$
\phi=\circledast_{\beta=0}^{N}\langle\text { bloom }[\beta]\rangle \circledast \circledast_{\gamma=k}^{N}\langle\operatorname{bin}[\gamma]\rangle
$$

To show that the loop invariant is preserved by the body, we can first show:

$$
\{\langle\operatorname{bloom}[n], \operatorname{bin}[n]\rangle\} u p d \leftarrow \operatorname{bloom}[n] \| \operatorname{bin}[n]\{u p d \sim \operatorname{bloom}[n] \| \operatorname{bin}[n]\}
$$

using RAssn. Noting that the boolean-or operator is a monotone operation, we may apply the frame rule NEGFrame to obtain:

$$
\{\langle\operatorname{bloom}[n], \operatorname{bin}[n]\rangle \circledast \eta\} u p d \leftarrow \operatorname{bloom}[n] \| \operatorname{bin}[n]\{\langle u p d\rangle \circledast \eta\}
$$

with the framing condition

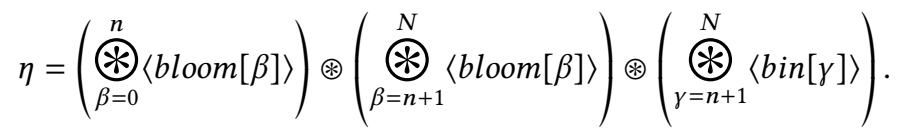

Thus, by re-associating the separating conjunction and applying RAssn for the remaining two assignments in the inner-most loop, we have:

$$
\{\phi\} \text { upd } \leftarrow \operatorname{bloom}[n] \| \operatorname{bin}[n] ; \operatorname{bloom}[n] \leftarrow u p d ; n \leftarrow n+1\{\phi\}
$$

and thus by Loop, we have:

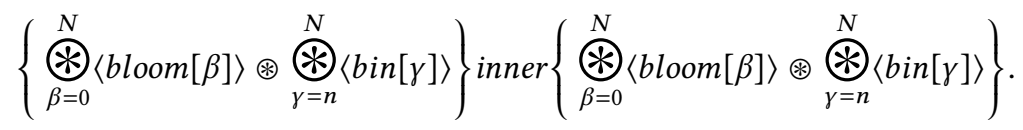

Now for loop mid, we establish the same loop invariant as we took before:

$$
\psi=\bigoplus_{\beta=0}^{N}\langle\operatorname{bloom}[\beta]\rangle
$$

If $\psi$ holds at the beginning of mid, then invariant for the inner-most loop $\phi$ holds after assigning 0 to $n$ and sampling bin, since bin is independent of $\psi\left(\mathrm{RSAMP}^{*}\right)$ and bin is distributed as $o h(n)$, which implies entries in bin are negatively associated (OH-PNA). Furthermore, $\phi$ implies $\psi$ at the exit of inner, by dropping the conjunct describing bin. Thus, $\psi$ is a valid invariant for mid, and the rest of the proof proceeds unchanged. 


\subsection{Permutation Hashing}

Our second example considers a scheme for hashing using a random permutation. Consider the program in Figure 7, from an algorithm for fast set intersection [Ding and König 2011]. Letting $B$ be the number of bins, and the data universe be $[B \cdot K]=\{1, \ldots, B \cdot K\}$ where $B \cdot K \geq N$, we first draw a uniformly random permutation $g$ of the data universe. Then, we hash the numbers $n \in[N]$ into $\operatorname{bin}[n]$ by applying the hash function $g$ and then taking the result modulo $B$. Then, we record whether the item landed in a specific bucket $Z$ by computing the indicator hit $Z[n]=[\operatorname{bin}[n]=Z]$, which is 1 if $\operatorname{bin}[n]=Z$ and 0 otherwise, and accumulate the result into the count $c t$.

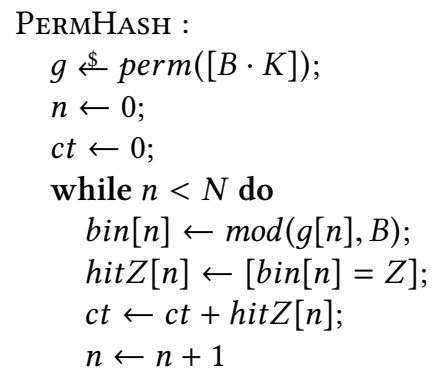

Fig. 7. Permutation Hashing

Our goal is to show that $c t$ is usually not far from its expected value, which is $N / B$. If the quantities $\{[\operatorname{bin}[n]=Z]\}_{n}$ were independent, we would be able to apply a standard concentration bound to the sum $c t$. However, $\{\operatorname{bin}[n]=Z\}_{n}$ are not independent: for instance, since exactly $K$ elements from $[B \cdot K]$ map to $Z$, if $\operatorname{bin}[n]=Z$ for $n \in\{0,1, \ldots, K-1\}$, then $\operatorname{bin}[K] \neq Z$.

Nevertheless, we can show that $\{[\operatorname{bin}[n]=Z]\}_{n}$ are negatively associated random variables. Intuitively, $\{g[n]\}_{n}$ are NA random variables because the result of a uniformly random permutation is NA. Then, $\{\operatorname{bin}[n]\}_{n}$ is computed by mapping the function $\bmod (-, B)$ over the array $g$; since this produces another uniform permutation distribution, the vector $\{\operatorname{bin}[n]\}_{n}$ is also NA. By similar reasoning $\{[\operatorname{bin}[n]=Z]\}_{n}$ is also NA, as it is obtained by mapping the function $[-=Z]$ over $\{\operatorname{bin}[n]\}_{n}$. Since this example is similar to the first Bloom filter example, except applying the negative association of the permutation distribution (Perm-PNA) and the permutation map axiom (Perm-Map), we defer the details to the appendix.

\subsection{Fully-Dynamic Dictionary}

For our next example, we consider a hashing scheme for a fully-dynamic dictionary, a space-efficient data structure that supports insertions, deletions, and membership queries. The top level of the data structure by Bercea and Even [2019] uses a two-level hashing scheme: elements are first hashed into a crate, and then hashed into a pocket dictionary within each crate. As part of the space analysis of their scheme, Bercea and Even [2019] proves a high-probability bound on the number of pocket dictionaries that overflow after a given number of elements are inserted.

We extract the program FDDicT in Figure 8a from the scheme in Bercea and Even [2019]. The program models the insertion of $N$ elements. Each element is first hashed into one of $C$ possible crates uniformly at random, and then hashed into one of $P$ possible pocket dictionaries uniformly at random. The variable $\operatorname{bin}[n]$ is a $C$ by $P$ matrix, with all entries zero except for the entry at (crate $[n]$, pocket $[n]$ ), which is set to 1 . Next, the program totals up the number of elements hashing to each (crate, pocket) pair, storing the result in the $C$ by $P$ matrix binCt. Finally, the program checks which (crate, pocket) pairs have count larger than some concrete threshold $T_{\text {bin }}$ (over), and totals up the number of full pocket dictionaries in each crate (overCt).

Our logic can prove a judgment of the following form:

$$
\{\top\} \operatorname{FDDict}\left\{\bigwedge_{\gamma=0}^{C} \operatorname{Pr}\left[\text { overCt }[\gamma]>P \cdot \rho_{\text {bin }}+T\left(\rho_{\text {over }}, P\right)\right] \leq \rho_{\text {over }}\right\},
$$

where the logical variables $\rho_{\text {bin }}$ and $\rho_{\text {over }}$ represents the parametric overflow properties. This formalizes a result similar to Bercea and Even [2019, Claim 21], which states that except with 


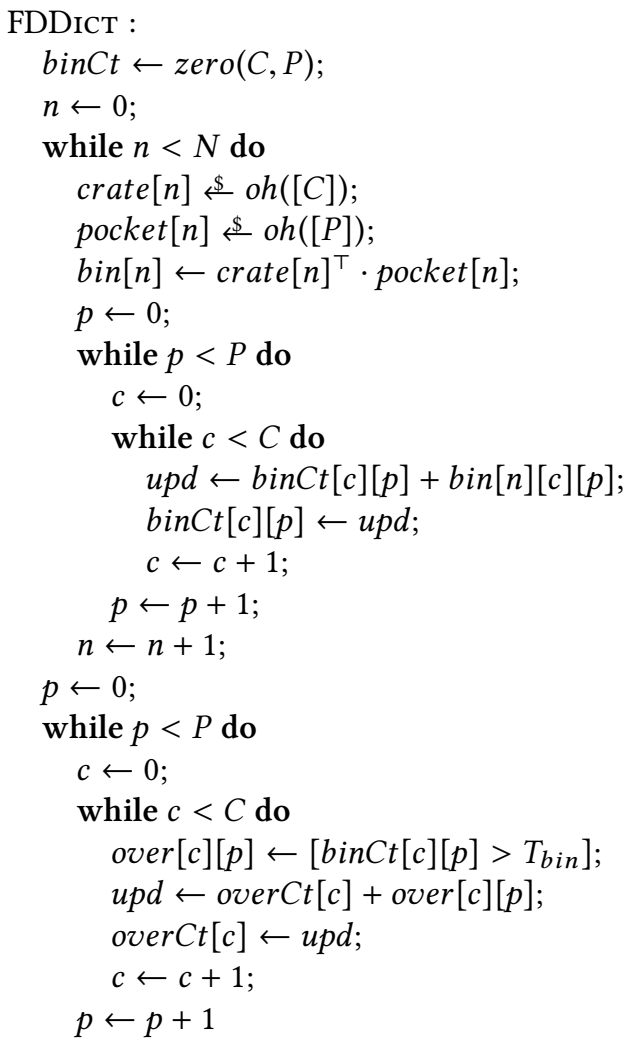

(a) Fully-dynamic dictionary [Bercea and Even 2019]

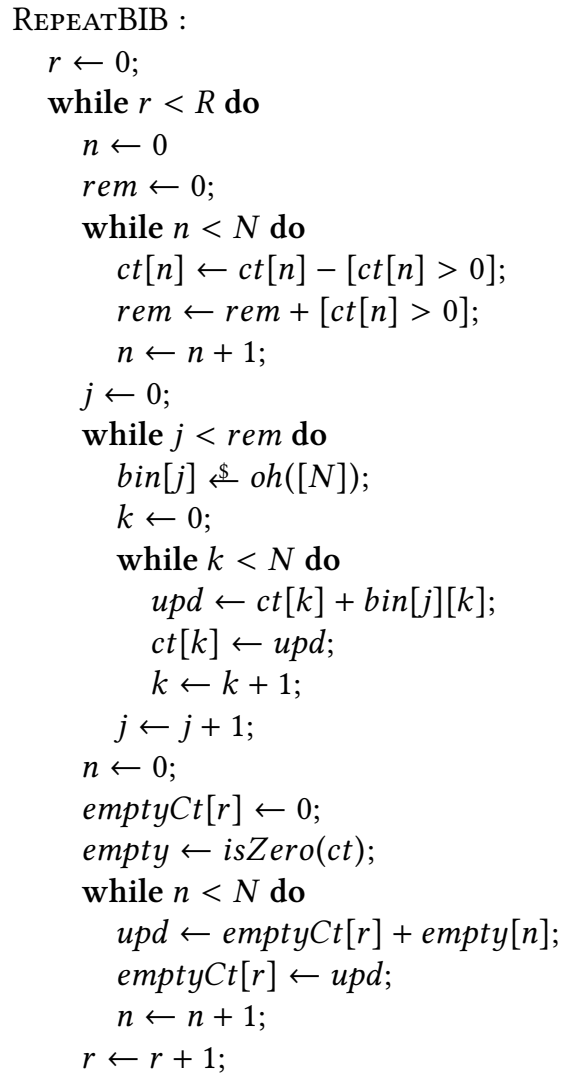

(b) Repeated balls-into-bins [Becchetti et al. 2019]

Fig. 8. Larger examples

probability $\beta$, all crates have at most $T_{\text {over }}$ overfull pocket dictionaries. The core of the proof shows that for every crate index $\gamma$, the counts $\operatorname{binCt}[\gamma][\beta]$ are negatively associated, using the NeGFrame rule as in the array version of the Bloom filter example. Then, we show that vector over $[\gamma][\beta]$, which indicates whether each pocket dictionary $\beta$ in crate $\gamma$ is overfull or not, is also negatively associated. This holds because over $[\gamma][\beta]$ is obtained from binCt $[\gamma][\beta]$ by applying a monotone function. Furthermore, the count of overflows over $C t[\gamma]$ is obtained by another monotone function on over $[\gamma][\beta]$ and thus its entries are also negatively associated.

\subsection{Repeated Balls-into-Bins Process}

Our final example considers a probabilistic protocol proposed by Becchetti et al. [2019], implemented as REPEATBIB in Figure 8b. Intuitively, the program implements a repeated balls-into-bins process. Initially, $N$ balls are distributed among $N$ bins $(c t[n])$. For $R$ rounds, in each round a ball is first removed from every non-empty bin. Then, the rem removed balls are randomly reassigned to bins. This process is useful for distributed protocols and scheduling algorithms, where the balls represent tasks and the bins represent computation nodes. Becchetti et al. [2019] proposed and analyzed this algorithm (e.g., bounding the maximum load, proving how long it takes for all balls to visit all bins). 
We can verify the following lower-bound on the number of empty bins, analogous to Becchetti et al. [2019, Lemma 1 and Lemma 2]:

$\left\{N \geq 2 \wedge \sum_{\alpha=0}^{N} c t[\alpha] \sim N\right\} \operatorname{RePEATBIB}\left\{\operatorname{Pr}\left[\bigvee_{\beta=0}^{R}\left(\operatorname{empty} C t[\beta]<N / 15-T\left(\rho_{\text {empty }}, N\right)\right)\right] \leq R \cdot \rho_{\text {empty }}\right\}$

Two aspects of this program make it more difficult to verify. First, there is a loop with a randomized guard: the number of removed balls rem is randomized quantity. Reasoning about such loops is challenging: our Loop rule is not directly applicable and only weaker rules are available for loops with general randomized guards. Becchetti et al. [2019] sidestep this problem by conditioning on the number of balls in each bin, which also fixes rem to be a deterministic value, proving the target property for every fixed setting, and then combining the proofs together. LINA can formalize this style of reasoning using the randomized case analysis rule (RCASE) to condition on rem's value, and then apply the Loop rule. However, the post-condition of RCASE must be closed under mixtures (CM), but independence and negative association are known not to satisfy this side-condition. Thus, it is not possible to prove negative association by first conditioning and then combining. To work around this second problem, we use a technique from Becchetti et al. [2019] and prove, on each conditional distribution, a high-probability bound using the Chernoff bound. High-probability bounds are CM, so we can apply RCASE to combine the results. The fact that LINA can handle this kind of subtle argument involving conditioning is a strength of our approach.

\section{RELATED WORK}

Bunched implications. The logic of bunched implications (BI) [O'Hearn and Pym 1999; Pym 2002] is a well-studied substructural logic. BI has a resource semantics [Pym et al. 2004], where states are resources and the separating conjunction combines compatible resources together. We follow Docherty's uniform presentation and investigation of BI [Docherty 2019]; in particular, our negative association model relies on Docherty's non-deterministic frame conditions, and we use his duality-theoretic framework to establish M-BI's metatheory.

Separation logics. The first separation logic was developed to verify pointer-manipulating programs [Ishtiaq and O'Hearn 2001; O'Hearn et al. 2001; Reynolds 2001]. There is long line of work on separation logic for concurrency, starting from [Brookes 2007; O'Hearn 2007] and continuing to the present day (e.g., [Jung et al. 2018; Sergey et al. 2015]).

More recently, separation logics have been developed for probabilistic programs. LINA is an extension of PSL [Barthe et al. 2020], a separation logic for probabilistic independence. Bao et al. [2021] propose DIBI, an extension of BI with a non-commutative conjunction, and developed a program logic with DIBI assertions that is capable of proving conditional independence. Batz et al. [2019] propose QSL, a separation logic where assertions have a quantitative interpretation, and used their logic to verify probabilistic and heap-manipulating programs. Tassarotti and Harper [2019] develop a separation logic for relational reasoning about probabilistic programs, using the coupling approach of pRHL [Barthe et al. 2012].

Verifying approximate data structures and applying concentration bounds. Bloom filters are a data structure supporting approximate membership queries (AMQs). Ceramist [Gopinathan and Sergey 2020] is a recent framework for verifying hash-based AMQ structures in the Coq theorem prover. Besides handling Bloom filters, Ceramist supports subtle proofs of correctness for many other AMQs. Compared with our approach, Ceramist proofs are more precise but also more intricate, applying theorems about Stirling numbers to bound the false positive probability. In contrast, our approach achieves a substantially simpler proof, albeit with less precise bounds. 
Prior works in verification have also applied the Chernoff bound to bound sums of independent random quantities (e.g., [Chakarov and Sankaranarayanan 2013; Wang et al. 2021]). While independence is easier to establish, the negative association property that we need is more subtle.

Negative dependence. There are multiple definitions of negative dependence in the literature, each with their own strengths and weaknesses. We work with negative association (NA) [Dubhashi and Ranjan 1998; Joag-Dev and Proschan 1983], because it holds in many situations where negative dependence should hold and it is closed under various notions of composition. Recently, the notion of Strong Rayleigh (SR) [Borcea et al. 2009] distribution has been proposed as an ideal definition of negative dependence. The SR condition satisfies more closure properties than NA does; in particular, it is preserved under various forms of conditioning. However, SR distributions have mostly been studied for Boolean variables only, and we do not know if an analogue of the monotone maps property of NA holds for SR.

Beyond theoretical investigations, negative dependence plays a useful role in many practical applications. In machine learning, negative dependence can help ensure diversity in predictions by a model [Kulesza and Taskar 2012], and fast algorithms are known to learn and sample from negatively-dependent distributions [Anari et al. 2016]. In algorithm design, negative dependence is a useful tool to randomly round solutions of linear programs to integral solutions [Srinivasan 2001]. Negative dependence can ensure that certain constraints are satisfied exactly after rounding, while still allowing concentration bounds to be applied to analyze the quality of the rounded solution.

\section{CONCLUSION AND FUTURE DIRECTION}

We introduced LINA, a probabilistic program logic that can reason about independence and negative association. Assertions in LINA are based on a novel probabilistic model of $M$-BI, an extension of the logic of Bunched Implications with multiple separating conjunctions. We demonstrated how to use LINA to reason about probabilistic hashing schemes, and a repeated balls-into-bins process. There are several natural directions for future work.

Other models of $M-B I$, and non-deterministic frames. The assertion logic $M$-BI was primarily motivated by our NA model, but it is general enough that we believe there are likely other natural models. Exploring these directions could allow modeling finer notions of separation, and could further justify $M-\mathrm{BI}$ as an interesting logic in its own right. It would also be interesting to see if there are other models that use a non-deterministic operator to combine resources, as proposed by Docherty [2019].

Verifying negative association for sampling algorithms. We used NA to analyze probabilistic hashing schemes. Another classical application of NA is in sampling schemes, which generate a sample from a target distribution while satisfying certain constraints [Brändén and Jonasson 2012; Dubhashi et al. 2007]. NA samplers are useful in algorithm design [Srinivasan 2001] and statistics, and it would be interesting to understand how to verify these programs. Many samplers employ rejection sampling, which is not easily analyzed in LINA but which could be expressed with an explicit conditioning operator, as in probabilistic programming languages [Gordon et al. 2014].

\section{ACKNOWLEDGMENTS}

We thank the anonymous reviewers for their helpful feedback and suggestions. This work benefited from discussions with Simon Docherty. This work was supported in part by the NSF under Grant No. 2035314, 1943130, 2040249, 2040222 and 2152831. 


\section{REFERENCES}

Nima Anari, Shayan Oveis Gharan, and Alireza Rezaei. 2016. Monte Carlo Markov chain algorithms for sampling Strongly Rayleigh distributions and determinantal point processes. In Conference on Computational Learning Theory (COLT), Vol. 49. Proceedings of Machine Learning Research, New York, New York, 103-115. http://proceedings.mlr.press/v49/anari16.html

Jialu Bao, Simon Docherty, Justin Hsu, and Alexandra Silva. 2021. A Bunched logic for conditional independence. In IEEE Symposium on Logic in Computer Science (LICS). IEEE, Rome, Italy, 1-14. https://doi.org/10.1109/LICS52264.2021.9470712

Jialu Bao, Marco Gaboardi, Justin Hsu, and Joseph Tassarotti. 2022. A Separation Logic for Negative Dependence. Proceedings of the ACM on Programming Languages 6, POPL (2022). https://arxiv.org/abs/2111.14917

Gilles Barthe, Benjamin Grégoire, and Santiago Zanella Béguelin. 2012. Probabilistic relational Hoare logics for computeraided security Proofs. In Mathematics of Program Construction (MPC). Springer, Madrid, Spain, 1-6. https://doi.org/10. 1007/978-3-642-31113-0_1

Gilles Barthe, Justin Hsu, and Kevin Liao. 2020. A probabilistic separation logic. Proceedings of the ACM on Programming Languages 4, POPL (2020), 55:1-55:30. https://doi.org/10.1145/3371123

Kevin Batz, Benjamin Lucien Kaminski, Joost-Pieter Katoen, Christoph Matheja, and Thomas Noll. 2019. Quantitative separation logic: a logic for reasoning about probabilistic pointer programs. Proceedings of the ACM on Programming Languages 3, POPL (2019), 34:1-34:29. https://doi.org/10.1145/3290347

Luca Becchetti, Andrea Clementi, Emanuele Natale, Francesco Pasquale, and Gustavo Posta. 2019. Self-stabilizing repeated balls-into-bins. Distributed Computing 32, 1 (2019), 59-68. https://doi.org/10.1007/s00446-017-0320-4

Ioana O. Bercea and Guy Even. 2019. Fully-dynamic space-efficient dictionaries and filters with constant number of memory accesses. CoRR abs/1911.05060 (2019). http://arxiv.org/abs/1911.05060

Burton H. Bloom. 1970. Space/time trade-offs in hash coding with allowable errors. Commun. ACM 13, 7 (1970), 422-426. https://doi.org/10.1145/362686.362692

Julius Borcea, Petter Brändén, and Thomas M. Liggett. 2009. Negative dependence and the geometry of polynomials. Journal of the American Mathematical Society 22, 2 (2009), 521-567. https://www.ams.org/journals/jams/2009-22-02/S0894-034708-00618-8/

Prosenjit Bose, Hua Guo, Evangelos Kranakis, Anil Maheshwari, Pat Morin, Jason Morrison, Michiel Smid, and Yihui Tang. 2008. On the false-positive rate of Bloom filters. Inform. Process. Lett. 108, 4 (2008), 210-213. https://doi.org/10.1016/j.ipl. 2008.05.018

Petter Brändén and Johan Jonasson. 2012. Negative dependence in sampling. Scandinavian fournal of Statistics 39, 4 (2012), 830-838. https://doi.org/10.1111/j.1467-9469.2011.00766.x

Stephen Brookes. 2007. A semantics for concurrent separation logic. Theoretical Computer Science 375, 1-3 (2007), 227-270. https://doi.org/10.1016/j.tcs.2006.12.034

Aleksandar Chakarov and Sriram Sankaranarayanan. 2013. Probabilistic program analysis with martingales. In International Conference on Computer Aided Verification (CAV). Springer, Saint Petersburg, Russia, 511-526. https://doi.org/10.1007/9783-642-39799-8_34

Bolin Ding and Arnd Christian König. 2011. Fast set intersection in memory. Proceedings of the VLDB Endowment 4, 4 (2011), 255-266. https://doi.org/10.14778/1938545.1938550

Simon Docherty. 2019. Bunched logics: a uniform approach. Ph. D. Dissertation. UCL (University College London).

Devdatt P. Dubhashi, Johan Jonasson, and Desh Ranjan. 2007. Positive influence and negative dependence. Combinatorics, Probability and Computing 16, 1 (2007), 29-41. https://doi.org/10.1017/S0963548306007772

Devdatt P. Dubhashi and Desh Ranjan. 1998. Balls and bins: A study in negative dependence. Random Structures and Algorithms 13, 2 (1998), 99-124. https://doi.org/10.5555/299633.299634

Kiran Gopinathan and Ilya Sergey. 2020. Certifying certainty and uncertainty in approximate membership query structures. In International Conference on Computer Aided Verification (CAV) (Lecture Notes in Computer Science, Vol. 12225). Springer, Los Angeles, California, 279-303. https://doi.org/10.1007/978-3-030-53291-8_16

Andrew D. Gordon, Thomas A. Henzinger, Aditya V. Nori, and Sriram K. Rajamani. 2014. Probabilistic programming. In Future of Software Engineering Proceedings (FOSE). Hyderabad, India, 167-181. https://doi.org/10.1145/2593882.2593900

Samin Ishtiaq and Peter W. O'Hearn. 2001. BI as an assertion language for mutable data structures. In ACM SIGPLAN-SIGACT Symposium on Principles of Programming Languages (POPL). London, England, 14-26. https://doi.org/10.1145/360204. 375719

Kumar Joag-Dev and Frank Proschan. 1983. Negative association of random variables with applications. The Annals of Statistics 11, 1 (1983), 286-295. https://doi.org/10.1214/aos/1176346079

Ralf Jung, Robbert Krebbers, Jacques-Henri Jourdan, Ales Bizjak, Lars Birkedal, and Derek Dreyer. 2018. Iris from the ground up: A modular foundation for higher-order concurrent separation logic. Fournal of Functional Programming 28 (2018), e20. https://doi.org/10.1017/S0956796818000151

Dexter Kozen. 1981. Semantics of probabilistic programs. F. Comput. System Sci. 22, 3 (1981), 328-350. https://doi.org/10 1016/0022-0000(81)90036-2 
Alex Kulesza and Ben Taskar. 2012. Determinantal point processes for machine learning. Foundations and Trends in Machine Learning 5, 2-3 (2012), 123-286. https://doi.org/10.1561/2200000044

Michael Mitzenmacher and Eli Upfal. 2005. Probability and computing - randomized algorithms and probabilistic analysis. Cambridge University Press.

Peter W. O’Hearn. 2007. Resources, concurrency, and local reasoning. Theoretical Computer Science 375, 1-3 (2007), $271-307$. https://doi.org/10.1016/j.tcs.2006.12.035

Peter W. O’Hearn and David J. Pym. 1999. The logic of bunched implications. Bulletin of Symbolic Logic (1999), 215-244 https://citeseerx.ist.psu.edu/viewdoc/download?doi=10.1.1.27.4742\&rep=rep1\&type=pdf

Peter W. O’Hearn, John C. Reynolds, and Hongseok Yang. 2001. Local reasoning about programs that alter data structures. In International Conference on Computer Science Logic (CSL) (Lecture Notes in Computer Science, Vol. 2142). Springer, Paris, France, 1-19. https://doi.org/10.1007/3-540-44802-0_1

Jens Pagel and Florian Zuleger. 2021. Strong-Separation Logic. In European Symposium on Programming (ESOP), Luxembourg City, Luxembourg (Lecture Notes in Computer Science, Vol. 12648). Springer, 664-692. https://doi.org/10.1007/978-3-03072019-3_24

Robin Pemantle. 2000. Towards a theory of negative dependence. F. Math. Phys. 41, 3 (2000), 1371-1390. https://doi.org/10. $1063 / 1.533200$

David J. Pym. 2002. The semantics and proof theory of the logic of Bunched implications. Applied Logic Series, Vol. 26. Kluwer Academic Publishers. Errata and Remarks maintained at: http://www.cantab.net/users/david.pym/BI-monographerrata.pdf.

David J. Pym, Peter W. O’Hearn, and Hongseok Yang. 2004. Possible worlds and resources: The semantics of BI. Theoretical Computer Science 315, 1 (2004), 257-305. https://www.sciencedirect.com/science/article/pii/S0304397503006248

John C. Reynolds. 2001. Intuitionistic reasoning about shared mutable data structure. Millennial Perspectives in Computer Science 2, 1 (2001), 303-321. https://citeseerx.ist.psu.edu/viewdoc/download?doi=10.1.1.11.5999\&rep=rep1\&type=pdf

Ilya Sergey, Aleksandar Nanevski, and Anindya Banerjee. 2015. Mechanized verification of fine-grained concurrent programs. In ACM SIGPLAN Conference on Programming Language Design and Implementation (PLDI). ACM Press, Portland, Oregon, 77-87. https://doi.org/10.1145/2737924.2737964

Aravind Srinivasan. 2001. Distributions on level-sets with applications to approximation algorithms. In IEEE Symposium on Foundations of Computer Science (FOCS). IEEE, Las Vegas, Nevada, 588-597. https://doi.org/10.1109/SFCS.2001.959935

Joseph Tassarotti and Robert Harper. 2019. A separation logic for concurrent randomized programs. Proceedings of the ACM on Programming Languages 3, POPL (2019), 64:1-64:30. https://doi.org/10.1145/3290377

Jinyi Wang, Yican Sun, Hongfei Fu, Krishnendu Chatterjee, and Amir Kafshdar Goharshady. 2021. Quantitative analysis of assertion violations in probabilistic programs. In ACM SIGPLAN Conference on Programming Language Design and Implementation (PLDI). ACM Press, Virtual, 1171-1186. https://doi.org/10.1145/3453483.3454102 